Research Article

\title{
Energy-Neutral Communication Protocol for Living-Tree Bioenergy-Powered Wireless Sensor Network
}

\author{
Yin Wu $\mathbb{D}^{1}{ }^{1}$ Bowen Li, ${ }^{1}$ Yongjun $\mathrm{Zhu},{ }^{2}$ and Wenbo Liu ${ }^{2}$ \\ ${ }^{1}$ Department of Information Science and Technology, Nanjing Forestry University, No. 159, Long Pan Road, \\ Nanjing 210037, China \\ ${ }^{2}$ College of Automation, Nanjing University of Aeronautics and Astronautics, No. 29, Jiangjun Avenue, Nanjing 211106, China
}

Correspondence should be addressed to Yin Wu; wuyin@njfu.edu.cn

Received 2 December 2017; Revised 19 March 2018; Accepted 10 April 2018; Published 10 May 2018

Academic Editor: Carlos T. Calafate

Copyright (c) 2018 Yin Wu et al. This is an open access article distributed under the Creative Commons Attribution License, which permits unrestricted use, distribution, and reproduction in any medium, provided the original work is properly cited.

\begin{abstract}
The purpose of this paper is to represent a living-tree biological energy powered wireless sensor system and introduce a novel energy aware MAC protocol based on remaining energy level, energy harvesting status, and application requirements. Conventional wireless sensor network (WSN) cannot have an infinite lifetime without battery recharge or replacement. Energy harvesting $(\mathrm{EH})$, from environmental energy sources, is a promising technology to provide sustainable powering for WSN. In this paper, a sensor network system has been developed which uses living-tree bioenergy as harvesting resource and super capacitor as energy storage. Moreover, by analyzing the power recharging, task arrangement, and energy consumption rate, a novel duty cyclebased energy-neutral MAC protocol is proposed. It dynamically optimizes each wireless sensor node's duty cycle to create a balanced, efficient, and continuous network. The scheme is implemented in a plant surface-mounted bioenergy power wireless sensor node system called PBN, which aims to monitoring the plant's growth parameters. The results show that the proposed MAC protocol can provide sustainable and reliable data transmission under ultralow and dynamic power inputs; it also significantly improves the latency and packet loss probability compared with other MAC protocols for EH-WSN.
\end{abstract}

\section{Introduction}

The limited available lifetime is a key bottleneck for most battery-powered wireless sensor networks (WSNs). Therefore, harvesting energy from the environment has been widely investigated to ensure the sustainability of WSN. As for this Energy Harvesting-WSN (EH-WSN), lots of studies have been carried out [1-3], the main research issue of energy consumption lies in two aspects: how to maximizing the harvested energy and how to maximizing the energy utilization efficiency, plus one research target: keep the EH-nodes stay in "energy neutral operation (ENO)" state [4]. Therefore, the hardware implementation and software communication protocols of EH-WSN are particularly attractive since they are just like the "body" and "nerve" of whole system. This paper proposes a novel MAC protocol with an energy harvesting prediction method to regulate the EH-node's work/sleep duty cycle based on the incoming power's changing status, residual energy level, and network task requirements. The aim is to make the system continuously operational in practical monitoring situations by minimizing the energy wastage and increasing the energy efficiency.

Recently, there are several works in the literature in which the authors used the duty-cycling technique to optimize EH-WSN's performance [5-9]: Yoo et al. proposed two novel dynamic duty cycle scheduling schemes (called DSR and DSP) in order to reduce sleep latency, while achieving balanced energy consumption among sensor nodes in EH-WSN [5]; Le et al. designed an efficient wakeup variation reduction power manager (WVR-PM) for wireless nodes powered by periodic energy sources, an energy-efficient synchronized wake-up interval MAC protocol (SyWiM) has also been proposed to solve the timing offset and clock drift issues [6]; Liu et al. have proposed a load and energy balancing receiver-initiated duty cycle MAC protocol (LEB-MAC) [7], it outperformed RI-MAC and DSR protocols in following aspects: low receiver and 
sender duty cycle, high throughput, high fairness, and low end-to-end delay; Bouachir et al. presented the EAMPAIDC protocol, an energy aware MAC protocol for $\mathrm{EH}-$ WSN based on individual duty cycle optimization [8]. It took into consideration nodes' residual energy and application and data requirements in order to define individual dynamic duty cycles so as to ensure continuous network operation; results showed that EAMP-AIDC protocol outperformed the IEEE 802.15.4 standard in terms of better energy consumption, increased survivability in energy savings, and in guaranteeing continuous operations. In particular, reference [9] proposed a duty cycle-based throughput optimal energy-neutral transmission work/sleep policy, then as well introduced a joint scheduling and routing protocol in multihop networks, aiming to provide network-wide packet communications under extremely limited node resources such as ultralow microbial fuel cell (MFC) power supply; results showed that it is able to provide sustainable and reliable data transmission under low and dynamic power inputs. With regard to EH-WSN of this specific kind, namely, using bioenergy as electrical power, researchers have also made quite a few contributions lately [10-13]: Zhang et al. compared two types of power management system (PMS) for MFC, charge pump capacitor converter type, and capacitor transformer converter type [10], They found that capacitor transformer converter type is recommended for ultralow MFC output and time-sensitive missions due to its wider input voltage range and shorter charging/discharging cycle; Erbay et al. proposed a PMS with dynamic maximum power point tracking capability; it could continuously detect the maximum power point (MPP) of the MFC and matches the load impedance of the PMS for maximum efficiency [11]; this PMS successfully powered a wireless temperature sensor that requires a voltage of $2.5 \mathrm{~V}$ and energy consumption of $85 \mathrm{~mW}$, and it could transmit the sensor data every $7.5 \mathrm{~min}$. Brunelli et al. presented the design of a battery-less monitoring system for plant health status, which exploits innovative Plant-MFC as joint power supply and biosensor for assessing the long-term health of the flora living in the surroundings [12], their bioelectrochemical system is used both as a power generator to supply the wireless embedded electronics and as a biosensor for estimating the status of the plant. Most recently Konstantopoulos et al. designed a selfpowered battery-less electric potential wireless sensor that harvests near-maximum energy from the avocado plant itself and transmits the signal tens of meters away; it has a total power consumption of $10.6 \mu \mathrm{W}$ and could accommodate simultaneous operation of multiple plants [13].

In the above designs, ambient bioenergy can be harvested and stored in the batteries or super-capacitors. However, using EH-sensor nodes also meet many challenges, such as the trade-off between idle listening, overhearing, and control packet overhead; the trade-off between energy-neutral operation and long latency; the extremely low recharging speed due to the typical feature of ultralow power in bioenergy; and the energy conversion efficiency is always a research focus under the situation of entirely "self" power supply. Our work intends to address these problems by inducing three advanced approaches: (i) incorporating an energy harvesting prediction algorithm in the proposed MAC protocol; (ii) adopting an optimized self-adaptive work/sleep duty cycle mechanism for every EH-node; and (iii) executing a differentiated access priority that considers both energy sustainability [14] and task requirement.

By considering these issues, we set to implement a livingtree biological energy powered wireless sensor system and design an novel "energy neutral" MAC protocol based on EH-node's remaining energy level, energy harvesting status, and application requirements. The optimization purposes are to maximize the total number of sampled data under energy harvesting constraints, as well as minimize the network transfer delay. To the best of our knowledge, this is the first time to analyze MAC protocol optimization in WSN powered by bioenergy. The main jobs and innovations are as follows:

(1) We employ a prediction approach in the node's duty cycle adjustment. The nodes could change their duty cycle intervals with fully aware of the future available energy. This procedure notably improves the accuracy and promptness of timing sequence in the nodes' duty cycle.

(2) The EH-WSN equipped with our novel algorithm can adapt to the variations of bioenergy in environment effectively and sensitively.

(3) We evaluate and compare the performance of our proposed MAC protocol with some aforementioned schemes. Results show that it outperforms the others in terms of throughput and latency.

The rest of this paper is organized as follows. In Section 2, the sensor node architecture and hardware component design are described. Section 3 explains our system model and describes the unresolved problem. In Section 4, the proposed MAC protocol and its mathematical explanations for sustainable sensing in a bioenergy-powered WSN are presented. Section 5 discusses the simulation and practical experiment results. In Section 6, we conclude the paper with a brief summary.

\section{PBN System Design}

Nowadays WSN has become a research hotspot in forest information monitoring field because of its miniaturization and integration [15-17]. Certainly, it still faces some difficulties due to the complexity and particularity in forestry application environment, especially the power supply problem: it is very troublesome to replace the batteries as conventional WSN always takes batteries as the energy storage unit. Hence, harvesting local environmental energy to settle this power supply issue is imperative. However, the present studies in EH-WSN mainly focus on the use of solar energy, wind energy, vibration energy, thermal energy, and electromagnetic energy [18], which all have defects in a practical forestry environment. The intensity of light in the forest is relatively weak and cannot be harvested during rainy and cloudy days; vibration does not exist in the woods in most conditions; wind velocity is small near the ground surface and 


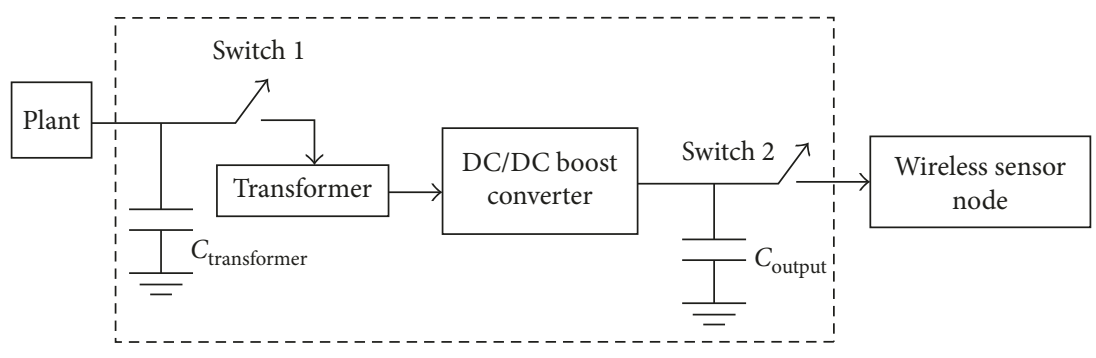

Figure 1: Schematic of proposed PBN.

is rather unstable; electromagnetic energy only exists close to the radiation emission station. Therefore, a more feasible energy harvesting method needs to be studied.

Recently, scientific explorations show that plants could become a potential source of bioenergy, and then this plant-bioenergy could be harvested to power small autonomous sensors (see above). Such EH-sensor does not need harsh working conditions and provides a new sight for EH-WSN in the forest. Accordingly, we conduct a research and design a prototype of plant surface-mounted bioenergy power wireless sensor node, with its block diagram shown in Figure 1.

The energy conversion circuit of PBN mainly consists of a voltage step-up converter. At the beginning, the first super-capacitor $C_{\text {transformer }}$ is charged by the low-voltage output of plant. Switch 1 is utilized to prevent the transformer and DC-DC converter drawing current from $C_{\text {transformer }}$ while it is being charged. Once the voltage of $C_{\text {transformer }}$ reaches the discharging voltage specified by the hardware, Switch 1 closes and $C_{\text {transformer }}$ works as the power source to drive the rest of the energy transfer circuits as well as the node. The voltage of $C_{\text {transformer }}$ is then amplified by the transformer. When $C_{\text {transformer }}$ discharges, the second super-capacitor $C_{\text {output }}$ starts being charged. Once the voltage of $C_{\text {transformer }}$ drops below the charging voltage, Switch 1 opens and $C_{\text {transformer }}$ begins being charged again. This process iterates for a few times until the voltage of $C_{\text {output }}$ reaches the required node voltage (such as $3.3 \mathrm{~V}$ for typical wireless sensors), when Switch 2 closes to power the node. The function of Switch 2 is to connect the node when $C_{\text {output }}$ is fully charged and to prevent the load from drawing current when $C_{\text {output }}$ is being charged.

We set the capacitance of $C_{\text {transformer }}$ to be $0.22 \mathrm{~F}$, and it was charged to $350 \mathrm{mV}$ and discharged to $100 \mathrm{mV}$. The average capacitor charging time was measured about 400 seconds. The super-capacitor $C_{\text {output }}$ was selected as $2.5 \mathrm{~F}$ in order to transmit data packets once fully charged. When $C_{\text {transformer }}$ was charged to $350 \mathrm{mV}$, Switch 1 closed and began to charge $C_{\text {output }}$. It took about $2.5 \mathrm{~h}$ to charge $C_{\text {output }}$ from $0 \mathrm{~V}$ to $3.3 \mathrm{~V}$. Then Switch 2 closed and started driving the wireless sensor node. When the voltage of $C_{\text {output }}$ decreased to $2.4 \mathrm{~V}$, Switch 2 opened and $C_{\text {output }}$ was then charged again. It took about $1 \mathrm{~h}$ to charge $C_{\text {output }}$ from $2.4 \mathrm{~V}$ to $3.3 \mathrm{~V}$. Actually we take the work/sleep mode toggle of wireless sensor node as Switch 2 because the power consumption in sleep mode is close to zero [19]. Hence, the charging procedure of $C_{\text {output }}$ can be always running. Figure 2 just shows the voltage variations of super-capacitor charging

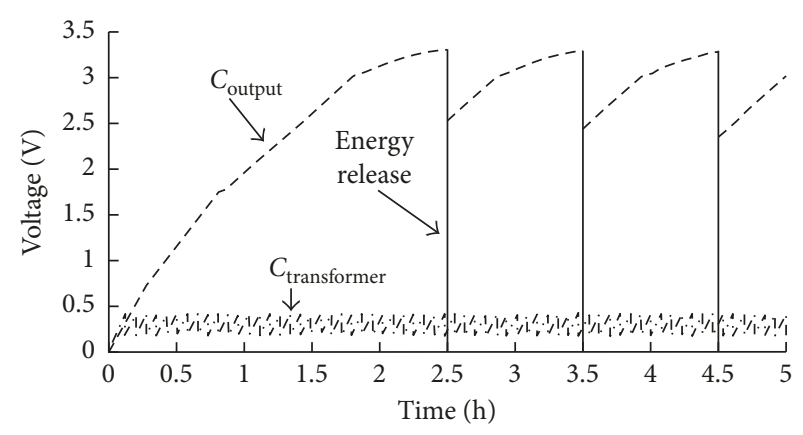

FIGURE 2: Changing statuses of super-capacitor voltage.

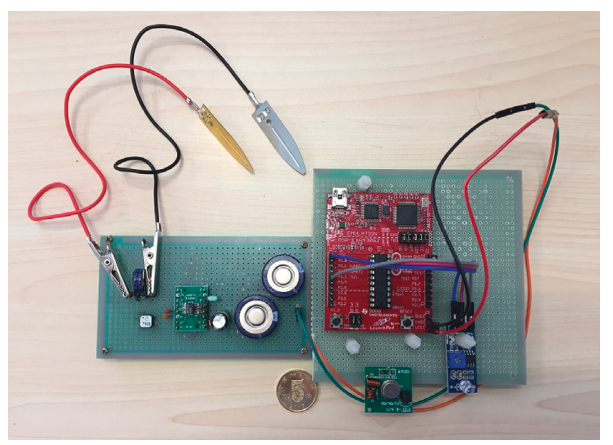

Figure 3: Illustration of proposed PBN.

process. In addition, the prototype of PBN is shown in Figure 3, an ultralow-power MSP430G2 is used as the control unit; a $315 \mathrm{MHz}$ super-regeneration wireless transfer module of $10 \mathrm{~mW}$ power consumption is adopted as communication unit, with a photosensitive sensor to acquire the illumination of plant.

\section{Problem Statement}

The purpose of this work is to research and evaluate a MAC protocol that solves the problems associated with PBN, which is powered by ultralow and varying bioenergy. Every PBN has a fixed number (denoted as $M$ ) of data packets to send. The data packet, with a size regarded as $Z$, contains illumination information of each plant. Specifically, our work thinks of a sensor network that is made up of plentiful PBNs deployed in a multihop mode. One PBN can both generate data by itself and receive data packets from other nodes while it acts as a relay. The sink is considered to own an unlimited power supply which could process and store 


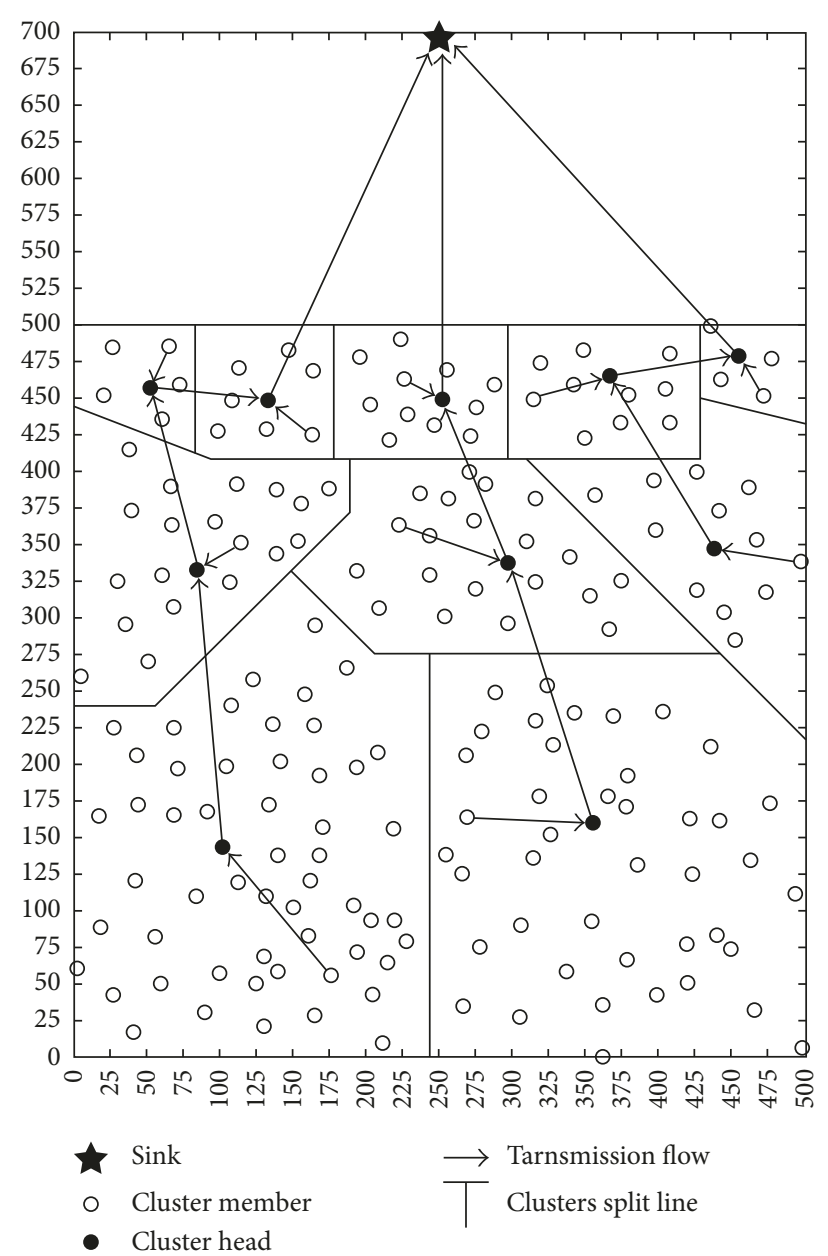

Figure 4: Operation structure of EHGUR-OAPR.

sensor data from all the nodes. In consideration of practical application, we set the network's routing protocol as a clustering structure, and our former research achievement EHGUR-OAPR [14] is carried on. EHGUC-OAPR is an unequal clustering routing protocol: clusters that are closer to the sink have smaller size. Apparently, it can balance the energy consumption of the entire network and improve the data delivery ratio. Then, MAC protocol in such clustering routing path is easier to understand: it takes the responsibility of access control in intracommunication between cluster head and its members, along with the intercommunication between cluster heads and the sink. On the contrary, the elections of cluster heads, the partition of cluster members, and the routing paths to the sink have already been calculated by routing algorithm. A corresponding network diagram is shown in Figure 4.

Because of the time-changing and ultralow-power supply, PBNs should work in a duty cycle fashion. The ideal scenario is that nodes only wake up when there are data waiting for transmission; otherwise, it falls into deep-sleep. This procedure is mainly under the control of MAC layer. Following the literature in [9], the MAC protocols for EHWSN can be classified into two categories: synchronous and asynchronous. The asynchronous type that adopts a receiver-initiated mechanism, which does not require any clock synchronization between sensor nodes, has been proven to outperform the state-of-the-art of traditional sender-initiated protocols and the synchronous protocols. Figure 5 just shows a basic operation of receiver-initiated communication between a sender and a receiver.

As shown above, whenever a receiver wakes up, it sends a wake-up beacon (WUB) and then waits for an incoming packet. Meanwhile, whenever a transmitter has a packet to send, it opens an idle listening window to receive a WUB from its receiver. As soon as a WUB is received, the transmitter performs Clear Channel Assessment (CCA), Calculation before Transmission (CBT) and then, forwards its Data Packet to the receiver. If a packet is successfully received, the receiver sends an Acknowledgment packet (ACK). Finally, both transmitter and receiver turn into sleep mode for energy saving.

With regard to the PBN, energy harvesting rate of each node changes according to many factors: sap concentration of the plant, seasonal variation, day-night rhythm, electrode material, solar irradiation, ambient temperature condition, and so on. The maximum generated power could vary from approximately $800 \mathrm{nW}$ to above $3000 \mathrm{nW}$ throughout the day [21]. As a result, this long and quickly changing power property of $\mathrm{PBN}$ poses a unique challenge to the existing duty cycle-based MAC protocols: neighboring nodes may never be able to wake up at a common interval to transmit data. Therefore, protocols for PBNs must be clearly understand of each plant's power characteristic and set their communications in an individual independent optimization strategy. Only this can guarantee a successful and practical communication of such system.

\section{Proposed MAC Protocol}

In this section, we present a novel asynchronous dutycycling energy-efficient MAC protocol called PB-MAC (plant-bioenergy MAC). PB-MAC achieves near-optimal energy efficiency both at receivers and at senders. In an optimal energy-efficient MAC protocol, when there is a packet to send, the sender and receiver wake up at the same time, transfer the packet reliably, and then both go to sleep again immediately. PB-MAC approaches this optimality in several ways: firstly, $\mathrm{PB}-\mathrm{MAC}$ is a receiver-initiated protocol but introduces the use of an individual energy harvesting prediction-based sequence to control each node's wakeup times; this allows the senders accurately predict the time at which a receiver will wake up. Thus, $\mathrm{PB}-\mathrm{MAC}$ reduces the duty cycle for receivers and senders both. Secondly, PBMAC calculates the transmission energy consumption of every PBN to seek for an optimal "ENO" access policy that gains the maximum throughput. This means that if the cluster head's bioenergy cannot afford a full-course data collection of each cluster member, it should strive for an optimum option that maximizes the network data throughput. Finally, PB-MAC could adjust packet transmission delay based on PBN's priority according to the task requirement. In other words, if a member node has an urgent message, then PB-MAC will try to improve its access priority to optimize delivery latency. 


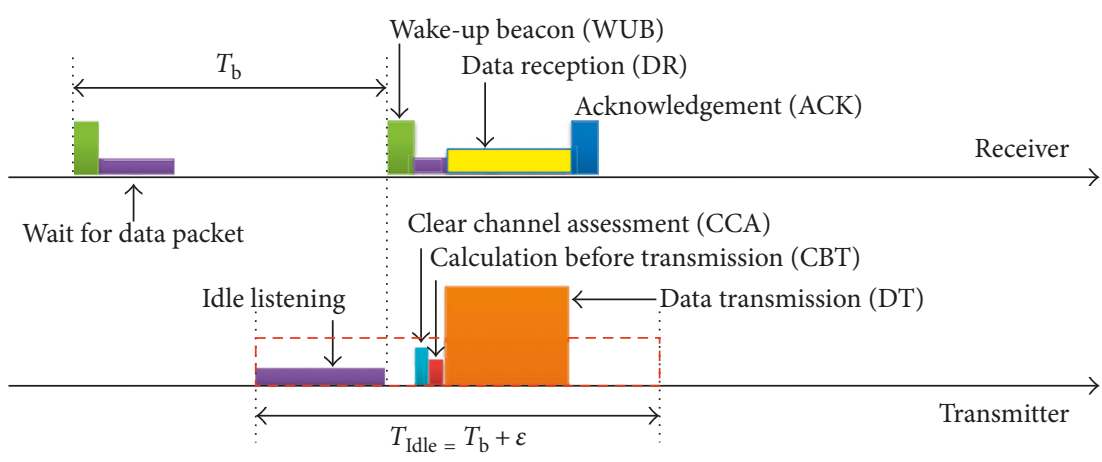

FIGURE 5: Basic communication scheme in RICER protocol [20].

The PB-MAC protocol operates in a time round structure, and the supposed round is divided into several time slots: each round has its own assignment individually, while the slots are used to apportion the working duty in a planned way at a detailed level. In this work, we use EH-round and $\mathrm{EH}$-slot for the energy management arrangement, whereas the MAC-round and MAC-slot for communication protocol scheme. Both two are associated by the energy consumption calculations.

4.1. Predictive-Wakeup Mechanism. An analysis of the plant as an energy source was conducted in [22, 23] for Pachira and bigleaf maple trees, respectively. It is concluded that plant-bioenergy is heavily influenced by environmental sunlight and moisture, and it approximately follows a periodic sinusoidal variation of 24 hours. Thus, we carried out a bioenergy voltage testing experiment during 3 days on a Koelreuteria in both summer time and late autumn, as shown in Figure 6. It is obvious that the records coincide with a day and night variation rhythm, as well as comply with a seasonal change. So we can have a energy harvesting prediction algorithm based on Weather-Conditioned Selective Additive Decomposition model (WCSAD): We first set the weather conditions of every day into three categories: sunny, rainy, and mixed; then we proceed to forecast the weather that only belongs to the same sort. In addition, we combine both the season variations during all the year and weather changes in one day together to calculate the energy that could be harvested in next time EH-rounds.

Firstly, the PBN needs to record the harvested energy $E_{\text {cal }}(d, s)$ in time EH-round $s$ of day $d$; hence, for the three categories there should be three matrixes $\left|E_{\text {cal }}(d, s)\right|_{D}$ that save $D$ days data independently. The average harvested energy in three kinds is

$$
\begin{aligned}
& \bar{E}_{\text {sunny }}=\frac{\sum_{i=1}^{D} \sum_{j=1}^{S} E_{\text {sunny }}(i, j)}{D}, \\
& \bar{E}_{\text {rainy }}=\frac{\sum_{i=1}^{D} \sum_{j=1}^{S} E_{\text {rainy }}(i, j)}{D}, \\
& \bar{E}_{\text {mix }}=\frac{\sum_{i=1}^{D} \sum_{j=1}^{S} E_{\text {mix }}(i, j)}{D},
\end{aligned}
$$

where $E_{\text {sunny }}, E_{\text {rainy }}$, and $E_{\text {mix }}$ are the harvested energy in sunny days, rainy days, and mixed days, respectively. Formula (1) should be updated every 24 hours to ensure the weather classification process. The detailed calculation rules are shown in Algorithm 1.

Here $\operatorname{Diff}(i, j)$ is a parameter concerns the seasonal changes:

$$
\begin{aligned}
\operatorname{Diff}(i, j)= & \beta\left[E_{\mathrm{cal}}(i-1, j)-E_{\mathrm{cal}}(i-2, j)\right] \\
& +(1-\beta)\left[E_{\mathrm{cal}}(i-2, j)-E_{\mathrm{cal}}(i-3, j)\right],
\end{aligned}
$$

where $\beta$ is just a tuning coefficient. After these corresponding classifications of former days, we can start to predict the up-coming energy, which would be harvested during time EH-round $s$ in the present day. The prediction value $E_{\text {est }}(i, j)$ equals

$$
\begin{aligned}
E_{\mathrm{est}}(i, j)= & E_{x}(i, j)+\alpha\left[E_{\mathrm{cal}}(i, j-1)-E_{x}(i, j-1)\right] \\
& +(1-\alpha)\left[E_{\mathrm{cal}}(i, j-2)-E_{x}(i, j-2)\right]
\end{aligned}
$$

where $\alpha$ is another adjustment coefficient. The latter two items in the above formula intend to represent the applicable harvesting energy's dynamic changes caused by different $\mathrm{EH}$ rounds. $E_{x}(i, j)$ is a weighted sum of corresponding rounds' harvested energy in the past reference days that belong to the same weather kind: $E_{x}(i, j)=\gamma E_{x-1}(i, j)+\gamma^{2} E_{x-2}(i, j)$. $\gamma$ is also a debug parameter. $E_{x-1}(i, j)$ just shows the corresponding rounds' energy in the last time with the same weather kind, in turn $E_{x-2}(i, j)$ is the day before last time within the same weather type.

By default, we can take the present day's weather type as the same as yesterday. However, a detecting mechanism is also designed to test the default hypothesis: we set up four testing points in a single day, and we compute the average harvested energy during the time from the beginning of day to the test point moment, if the value has a great difference with the last day's data, we should regulate the weather type immediately.

4.2. Throughout Optimization Mechanism. Here, in this part, a duty cycle-based energy-neutral MAC protocol is proposed to accomplish an optimal throughput and appreciable quality of service. By studying on CSMA/CA protocols and 


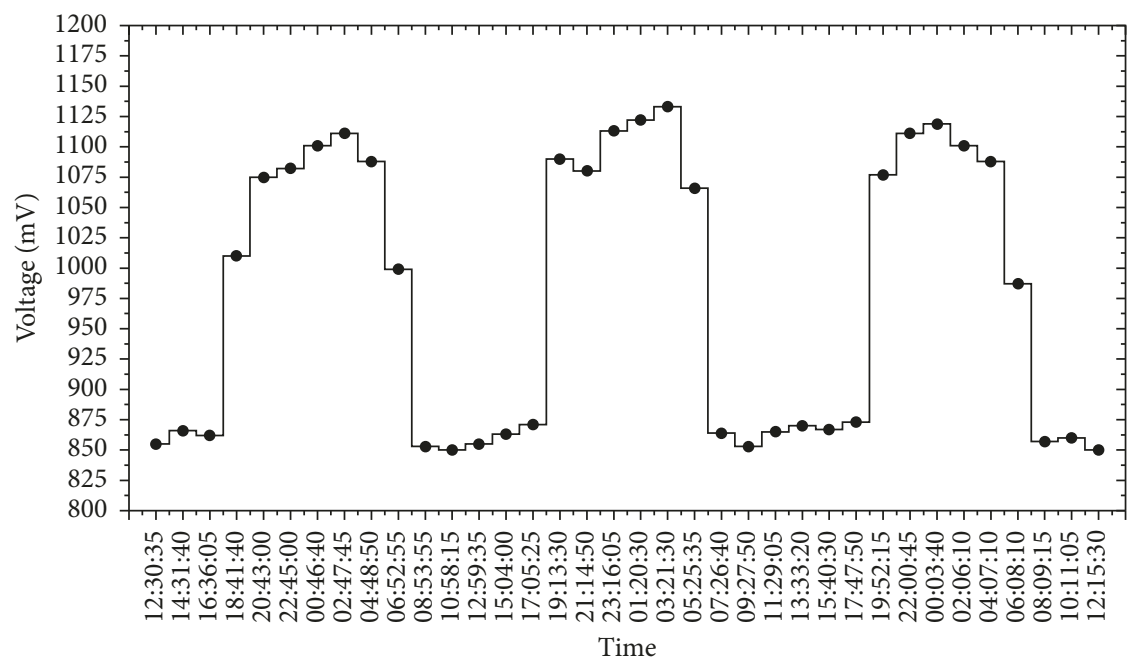

(a)

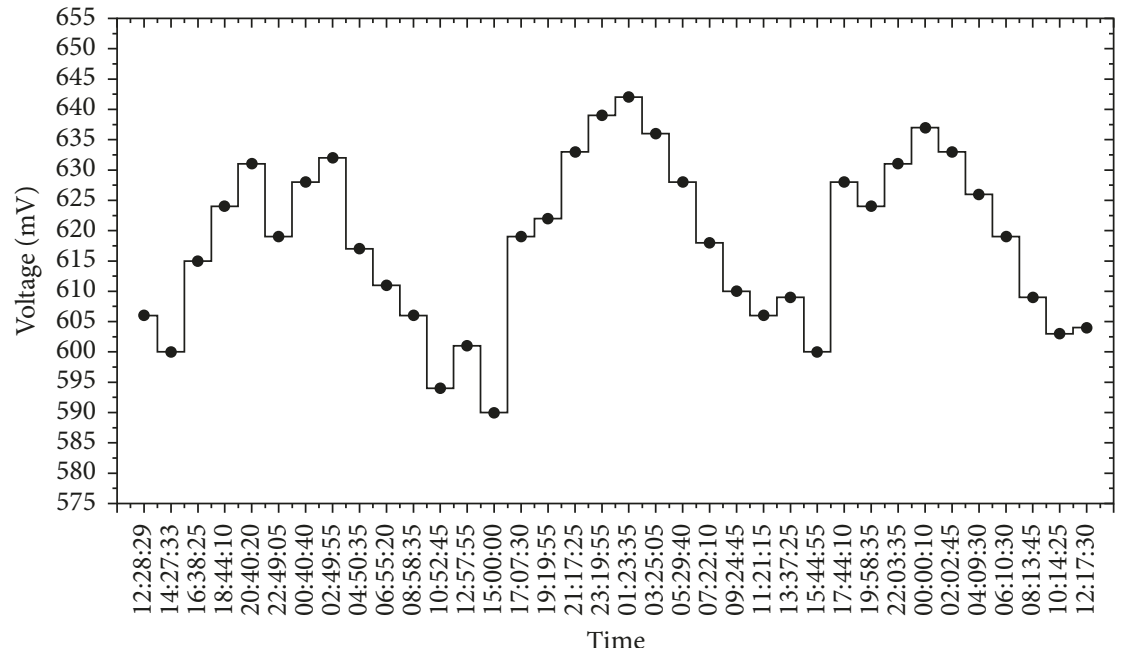

(b)

Figure 6: Bioelectric voltage variation of a Koelreuteria: (a) in summer time (b) in late autumn.

(1) BEGIN

(2) If $\left(\sum_{j=1}^{S} E(i, j)>(1+\right.$ Diff $\left.) \bar{E}_{\text {rainy }}\right)$

(3) $\quad$ day $i$ is a rainy day, update $E_{\text {rainy }}$ \}

(4) Else if $\left(\sum_{j=1}^{S} E(i, j)<(1-\right.$ Diff $\left.) \bar{E}_{\text {sunny }}\right)$

(5) $\quad$ day $i$ is a sunny day, update $\left.E_{\text {sunny }}\right\}$

(6) Else

(7) $\quad$ day $i$ is a mixed day, update $\left.E_{\text {mix }}\right\}$

(8) END

Algorithm 1: Harvested energy classification algorithm for different weather conditions.

TDMA protocols, we found that CSMA/CA protocol easily leads to serious conflicts, thus causing heavy package loss and TDMA protocol likely leads to large delay. Therefore, we try to combine CSMA/CA protocol and TDMA protocol together reasonably to design a MAC protocol that conforms to the PBN scenario. The hybrid
TDMA/CSMA protocol, that is, PB-MAC, adopts TDMA mode at intracluster communication stage, while performs CSMA/CA mode at intercluster communication stage. The detailed time sequence strategy is described in the following section.

4.2.1. Initialization Phase. In this phase, all the PBNs should be set-up on the living trees and equipped with an initial energy $B_{\text {initial }}$ randomly. After that, every node transmits parameters such as its distances information and energyharvesting rate directly to the sink and then the sink will run clustering algorithm to elect the cluster heads and members, receptively. Finally, this computational result will be downloaded to each node, namely, the routing procedure has been fulfilled [14].

Hence, the cluster member nodes should transmit messages to their corresponding cluster head to acknowledge the transmission arrangement, which is the next phase. 
4.2.2. Intracluster Phase I. In this phase, the cluster members must establish communication connection with their cluster head by the CSMA/CA mode. Firstly, the member node $i$ sends a request frame RTS before sending the data packet, and after receiving the RTS, the cluster head sends CTS to reply. Then node $i$ sends its own information to the head node such as the ID number, amount of data it collects, energy harvesting rate, and predicting result. After receiving this packet, the head node should send an ACK packet that contains its wakeup sequence information to the node $i$. All the other nodes in the cluster will listen to the channel and wait until it is clear, then they would go on completing for the channel and repeat the process until all communications have completed.

4.2.3. Intracluster Phase II. By using the CSMA/CA mechanism, the cluster head collects all member nodes' preliminary information; thus, the intracluster transmission proceeds to the next scheduling access stage, which uses TDMA mechanism. We design an optimal access-scheduling algorithm to allocate the time MAC-slots for both maximum throughput and balanced network load (Algorithm 2).

In formula in Algorithm 2, $E_{M, n}$ means the maximum energy storage capacity of the node $n, E_{S, n}$ is the current stored energy in node $n, P_{E H, n}>0$ is the individual harvesting power rate of node $n$, and $\sigma, \mu$ are appropriately chosen constants. We use ST $(n)$ to evaluate PBN's energy sustainability. The node with higher value is more patient than the node with lower value in transmitting packets; in other words, the low energy sustainability node is more desperate to transmit data before all the energy is discharged. So, in this context, the access probability can be determined to be inversely proportional to the energy sustainability.

Next, the cluster head should check if there is request to relay other head nodes' packets or transmit its own packet, as well as predict the forthcoming energy that can be harvested. Based on these above information, access allocation algorithm will calculate the number of nodes that could be visited:

$$
\begin{aligned}
E_{S, n}+E_{\mathrm{est}}(i, j)= & I[a(z)] \cdot E_{\mathrm{TX}}(z)+I[b(z)] \cdot E_{\mathrm{RX}}(z) \\
& +I[n 1] \cdot E_{\mathrm{RX}}(n 1)+I[n 2] \cdot E_{\mathrm{RX}}(n 2)+\cdots,
\end{aligned}
$$

where $E_{\mathrm{TX}}(z), E_{\mathrm{RX}}(z)$ are the energy consumptions in the cluster head node $Z$ 's data transmission and reception, respectively. $E_{\mathrm{RX}}(n i)(i=1,2,3, \ldots)$ is the data reception energy consumption for cluster member node $i . I(\cdot)$ is the indicator function and $a(z), b(z)$ are the events that cluster head transmits and receives packets with other cluster heads; besides $n i$ is the event that cluster member $i$ transmits data to the head node. Apparently, the head node would obtain the exact number of executable nodes and broadcast the accurate access-scheduling time to them, thus performing the data communication in a TDMA mode. As for all the executable nodes, when they receive the broadcast message from the head node, they should analyze the schedule time based on their energy status: if matching with its work/sleep duty cycle, the member node will send an ACK message back; in contrast, it will send a NACK message, and the head node should postpone to next one and adjust the scheduling plan.

Finally, the cluster head collects member nodes' packets and needs to decide whether to transmit these data forward or not. Here, we choose to use a random number generator with range from 0 to 1 : if the generated number exceeds a specified threshold $\Omega$, then it starts to prepare for the data transmission with next hop; otherwise, the head node sleeps immediately. Certainly here exists a head node-updating program, sink should collect every cluster nodes' energy information and compute an optimal election result. The whole operation diagram is shown below in Figure 7.

4.2.4. Intercluster Phase. After completing the data acquisition task in the cluster heads, it needs to decide how and when to transmit the packets to the sink. Based on the former phase, the head node could set the occasion of intercluster communication clearly. However, it should be noted that if the head node decides to transfer packet to the next hop, the actual sending process would take place in the next time MAC-round, and the current head node only transmits an appointment to the next hop at the present MAC-round. This procedure enhances the transmission reliability considerably and does not cause obvious time delay.

Therefore, all the cluster head nodes transmit packets accordingly based on the sequence of routing protocol. In addition, we set intercluster transmission in a senderinitiated way [24] to improve the energy efficiency, just as shown in Figure 8.

4.3. Priority Optimization Mechanism. In this section, we consider applications that require some $\mathrm{PB}$ nodes to transfer critical or urgent data to the sink as soon as possible from the time they are generated. The source node and all relay nodes between the source and destination should send these urgent data packets faster than normal data packets. Using an additional priority optimization mechanism, the described PB-MAC could specify these urgent data as high priority data packets and transmit these data packets first.

Here we modify the cluster head's access-scheduling algorithm with a packet priority value. In other words, time MAC-slots are allocated by both the energy sustainability of cluster member node and its packet priority; the new access order during the TDMA phase is determined by the sum of two parameters:

$$
N_{\text {Total }}=\chi \cdot N_{\mathrm{ES}}+(1-\chi) \cdot N_{\mathrm{P}} \text {, }
$$

where $N_{\text {Total }}$ is the optimized sequence in the subsequent access allocation algorithm, $\chi$ is the coefficient range from 0 to $1, N_{\mathrm{ES}}$ is the order of energy sustainability, and $N_{\mathrm{P}}$ is the order of packet priority. Using this priority control function, the sender nodes can notify their packet priority to the cluster head node. In addition, after receiving these senders' 

(1) BEGIN
(2) Calculate member node $n$ 's energy sustainability $\mathrm{ST}(n)=\left(E_{M, n} /\left(\left(P_{\mathrm{EH}, n}+\sigma\right) \log \mu\right)\right)\left(\mu^{\lambda(n)}-1\right)$, where $\lambda(n)=\left(E_{M, n}-E_{S, n}\right) / E_{M, n}$
(3) Judge if there exists inter-cluster communications on this cluster head node
(4) Predict the energy that can be harvested in this time round
(5) Sort the list of all the member nodes' ST $(n)$ by ascending counts
(6) Allocate the time slot by the above list
(7) Compute the energy budget formulas; determine the total number of nodes that can be accessed
(8) Communicate with each cluster members according to the above result
(9) Decide whether to transmit the collected data to the next hop cluster head or not
(10) Decide whether to change the current cluster head node or not
(11) END

Algorithm 2: Access-scheduling optimization algorithm in the intracluster phase.

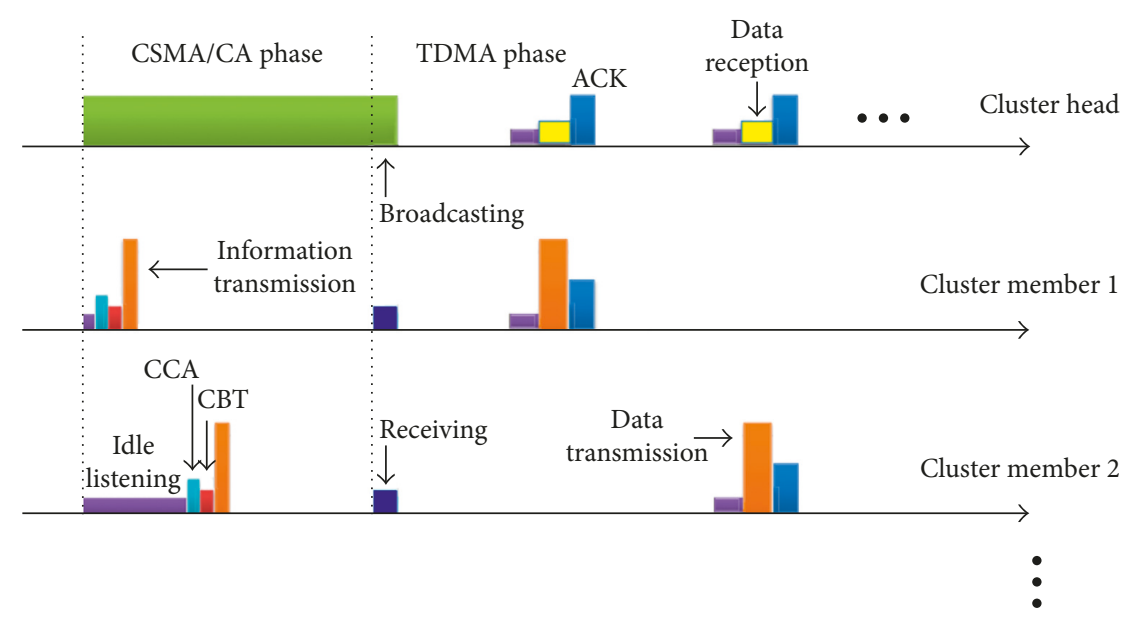

FIGURE 7: Intracluster work/sleep duty cycle diagram (note that the receiver and transmitter diagram are of very different time scales).

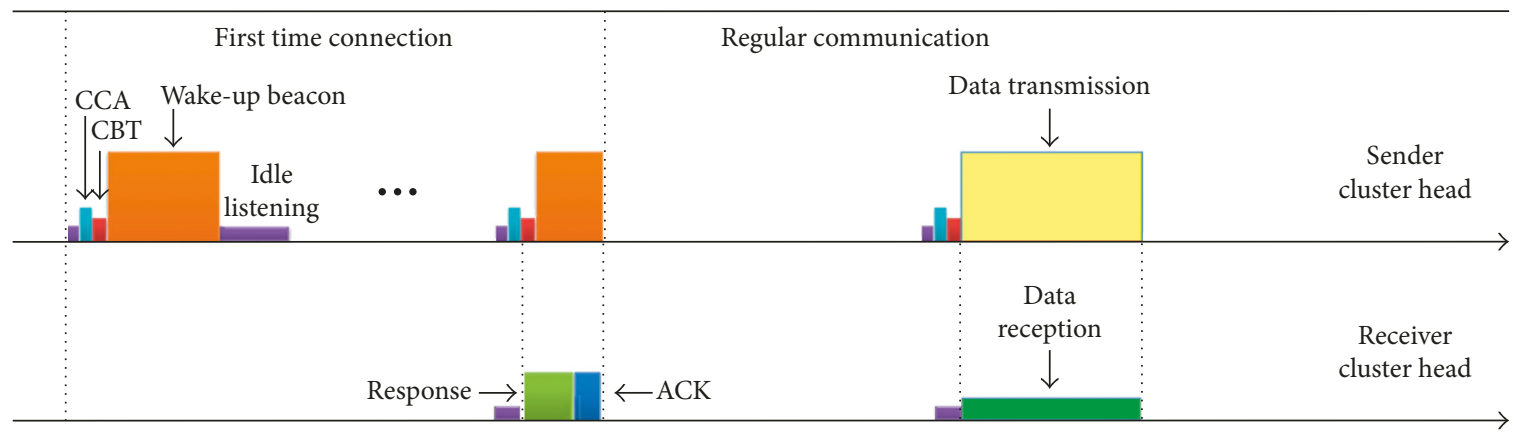

FIGURE 8: Intercluster work/sleep duty cycle diagram.

packets based on priority, the head node also must adjust its intercluster communication probability to transfer the data as soon as possible: it should start the intercluster transmission immediately and make an appointment with its next hop node.

As a result, $\mathrm{PB}-\mathrm{MAC}$ could predict the harvested energy of next time round, calculate an optimal access sequence of the cluster member to keep all nodes under "ENO" state; maximize the data throughput based on the hybrid TDMA/CSMA transfer mode and minimize the transmission delay based on the priority technique. A detailed duty cycle timing sequence along with the energy harvesting is described in Figure 9 for instance:

As shown above, all cluster nodes work in a duty cycle mode; they calculate the harvested power and turn down to sleep whenever the energy is insufficient. Due to the individual energy-harvesting rate, the working period of every member node may be different. They would follow the working sequence based on the proposed approach and carry out the data-sampling task as well. In turn, cluster heads take charge of gathering all the data and then send to the sink. Next, we will evaluate and compare the PB-MAC with some references. 


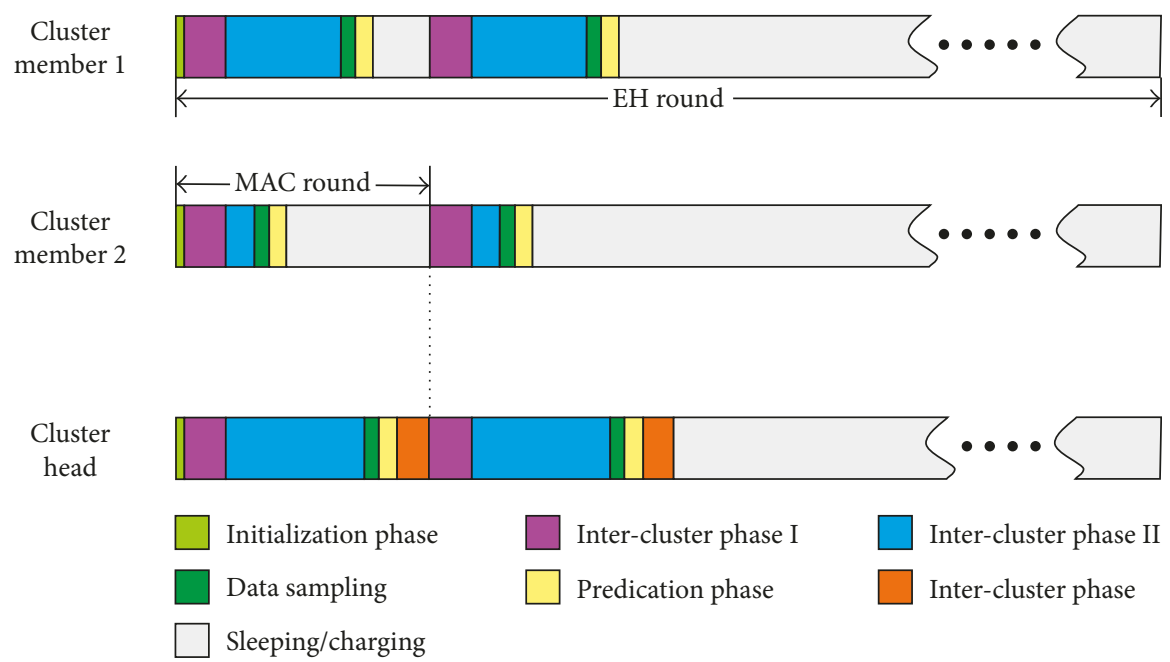

FIgURE 9: The overall duty cycle diagram of nodes in one cluster.

\section{Performance of PB-MAC}

To validate the proposed PB-MAC and evaluate its performance, we conduct simulation studies using OMNeT++. The simulated network is a $500 \mathrm{~m} \times 500 \mathrm{~m}$ square area with total 200 randomly deployed nodes (Figure 4 shows the network topology). Key parameter values are listed in Table 1.

Here, we adopt the energy consumption model in [25]: $E_{\mathrm{TX}}(k, d)$ means the transmission energy of transferring $k$ bits for distance $d$ and $E_{\mathrm{RX}}(k)$ just means the reception energy of receiving $k$ bits.

$$
\begin{aligned}
E_{\mathrm{TX}}(k, d) & = \begin{cases}E_{\text {elec }} k+E_{\mathrm{fs}} k d^{2}, & \text { Intracluster, } \\
E_{\text {elec }} k+E_{\mathrm{mp}} k d^{4}, & \text { Intercluster, }\end{cases} \\
E_{\mathrm{RX}}(k) & =E_{\text {elec }} k .
\end{aligned}
$$

We compare PB-MAC against RI-MAC with DSP (denoted as RI-DSP) [5], EAMP-AIDC [8], and MFC-MAC [9], all operating under the plant-bioenergy-powered circumstance [13]. Firstly, we study the protocols using the same network settings as in Figure 4 and an initial energy level for all sensors to be $30 \%$, we also assume that all sensors undertake equal power harvesting rate and there are three different scenarios, which are $0.25 \mathrm{~mW}, 0.75 \mathrm{~mW}$ and $1.25 \mathrm{~mW}$, respectively. The results of a 24-hour simulation run are presented in Figure 10.

The results shown in Figure 10 are aimed at evaluating the functionality of PB-MAC. Since the initial energy level is set at $30 \%$, there may be fewer nodes active, more network holes, and retransmissions. While $\mathrm{PB}-\mathrm{MAC}$ shows the best overall performance, we note that MFC-MAC's probeminimization mechanism was able to keep the senders' duty cycles short, saving significant energy. Longer sender duty cycles (Figure 10(b)) of RI-DSP and EAMP-AIDC manifest higher energy usage, more network holes as nodes need more sleep cycles, and consequently, higher node-to-sink delays (Figure 10(a)). All these protocols consume their energy storage quickly (Figure 10(c)) but will also replenish to different extent thereafter. Starting with
TABle 1: Key parameter values.

\begin{tabular}{lc}
\hline Parameter & Value \\
\hline Network monitoring area & $(0,0) \sim(500,500)$ \\
Coordinate of sink & $(250,700)$ \\
$\sigma, \mu$ & $0.1,5$ \\
$\alpha, \beta, \gamma$ & $0.5,0.6,0.62$ \\
$D$ & 5 \\
EH-round & $1 \mathrm{hour}$ \\
$\mathrm{MAC}$-round & $12 \mathrm{~second}$ \\
$Z$ & $32 \mathrm{bit}$ \\
$E_{\mathrm{elec}}$ & $50 \mathrm{~nJ} / \mathrm{bit}^{-2}$ \\
$E_{\mathrm{fs}}$ & $10 \mathrm{pJ} /\left(\mathrm{bit} \cdot \mathrm{m}^{-2}\right)$ \\
$E_{\mathrm{mp}}$ & $0.0013 \mathrm{pJ} /\left(\mathrm{bit} \cdot \mathrm{m}^{-4} \mathrm{~s}\right)$ \\
$E_{M, n}$ & $10 \mathrm{joule}$ \\
$\chi$ & 0.382 \\
EH-slot & $12 \mathrm{minute}$ \\
$\mathrm{MAC}$-slot & $3 \mathrm{~second}$ \\
$M$ & 4 \\
\hline
\end{tabular}

$30 \%$ energy level puts many nodes in longer sleep cycles and leaves the network with dynamic routes, and this increases the packet loss probability among data flows. While both PBMAC and MFC-MAC are able to achieve higher packet delivery ratio (PDR) than the other protocols, PB-MAC performs better and maintains $100 \%$ PDR under extremely limited energy harvesting conditions as shown in Figure $10(\mathrm{~d})$. Therefore, in this particular plant-bioenergy scenario, the energy-harvesting rate is too low for the other three protocols, and only $\mathrm{PB}-\mathrm{MAC}$ is able to continue operating and keep the energy level above 30\% (note: for the convenience of analysis and comparison, all the prediction mechanisms in the above protocols are set full success due to the uniform energy harvesting rate, as well all data packets have the same priority).

Moreover, we adjust the position of sink $(250,700)$ along a straight line to the coordinate $(250,500)$, and we record two network quality indexes of PB-MAC likewise: the average node-to-sink latency and the node average remaining energy. The result is shown below in Figure 11. Here the energy-harvesting rate is assumed $0.75 \mathrm{~mW}$. Obviously, we 


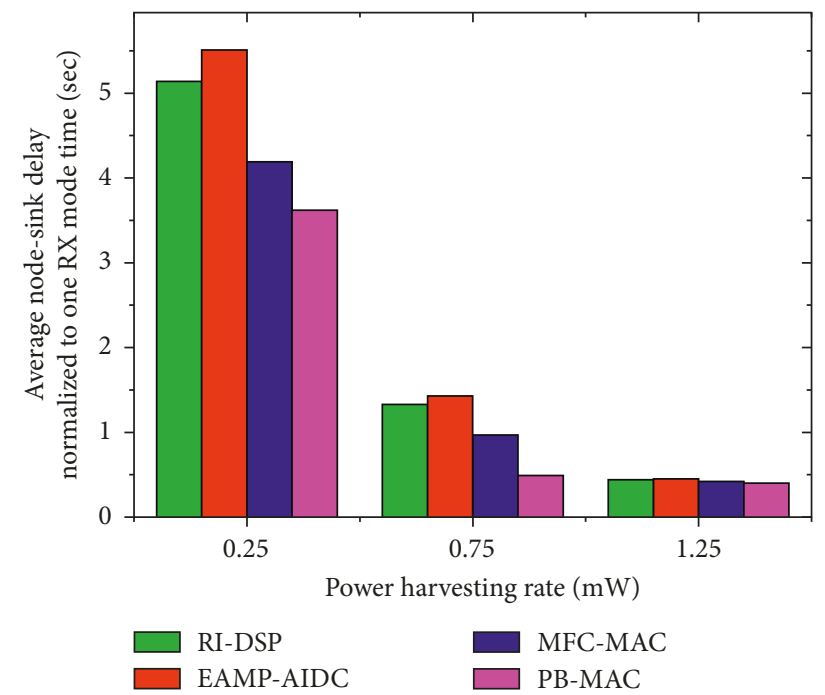

(a)

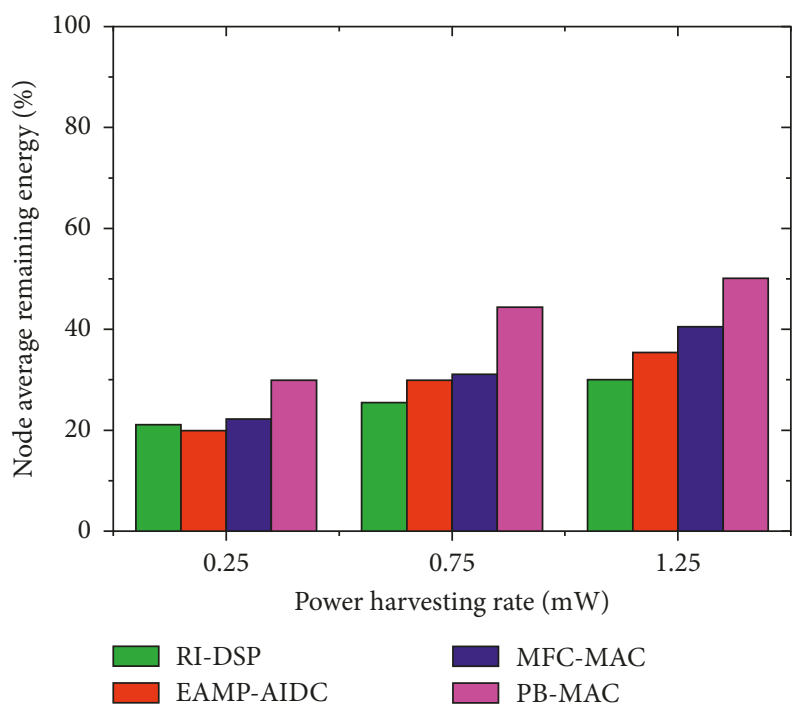

(c)

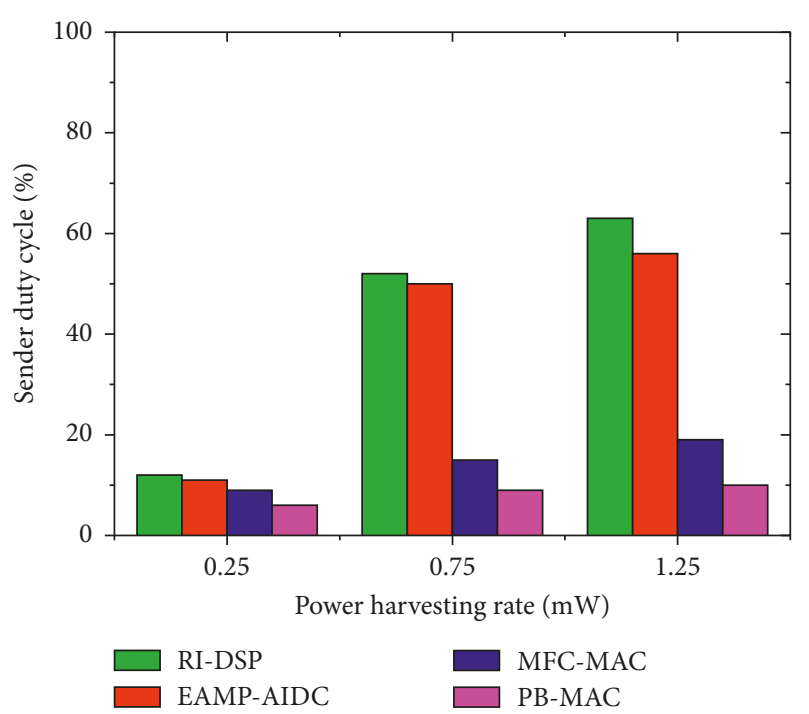

(b)

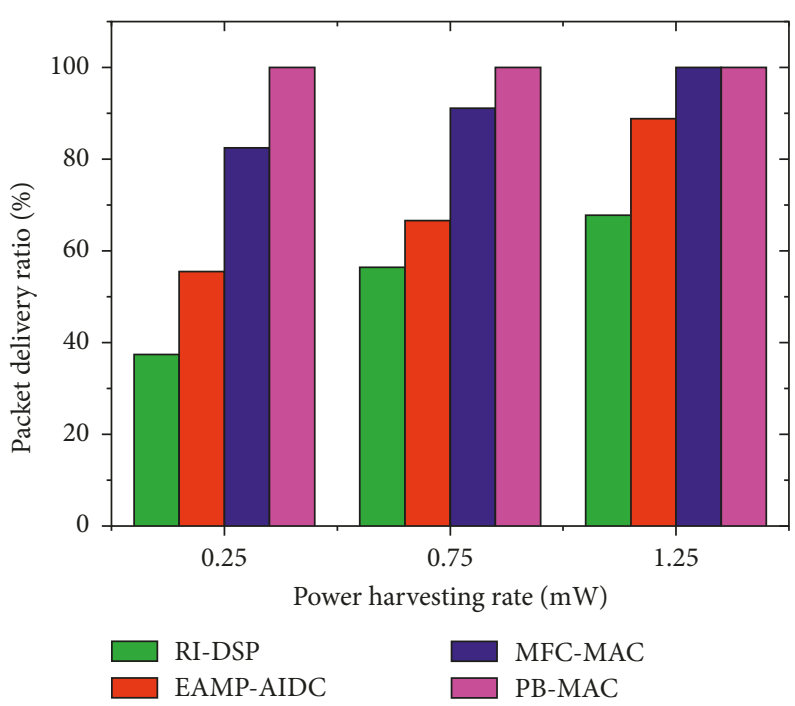

(d)

Figure 10: RI-DSP, EAMP-AIDC, MFC-MAC, and PB-MAC performance, with 30\% initial energy level. (a) Average node-to-sink latency versus power harvesting rate, (b) sender duty cycle versus power harvesting rate, (c) node average remaining energy versus power harvesting rate, (d) packet delivery ratio versus power harvesting rate.

can see from the diagram that node average remaining energy gets greater when the sink moves towards the PBNs and the average node-to-sink latency decreases conversely. The reason is when the average distance between the sink and nodes becomes smaller, the transmission energy consumption also diminishes accordingly; meanwhile, the work/sleep duty cycle is optimized, and the improved operating time reduces the average latency.

Secondly, we use realistic data of our proposed PBNs measured at a mingled forest with area $50 \mathrm{~m} \times 50 \mathrm{~m}$ (including Koelreuteria, cedar, birch, etc.) over 7 days in March 2018, starting at early morning, all 30 PBNs having an initial energy level of $60 \%$. Nodes are placed in the field with a random uniform distribution while the sink is located at the center, and a random cluster member node has been chosen to analysis specifically, and some of the related results are shown below:

As expected, the member nodes equipped with PB-MAC are able to harvest bioenergy systematically through the day and dissipate its energy supply rapidly. Nevertheless, by 17 : 30 on the fifth day, the node starts to replenish its energy supply quickly (Figure 12(a)) and continue harvesting enough energy to operate: this is due to the precise operation of prediction algorithm. With the adequate storage, node is able to stay active longer, producing more data packets to the sink. This improves the data throughput and enhance the network performance in general (Figures 12(c) and 12(d)), especially based on the packets with randomly assigned priority (3 level), PDR is approximates to $100 \%$ over the latter testing period (Figure 12(b)). 


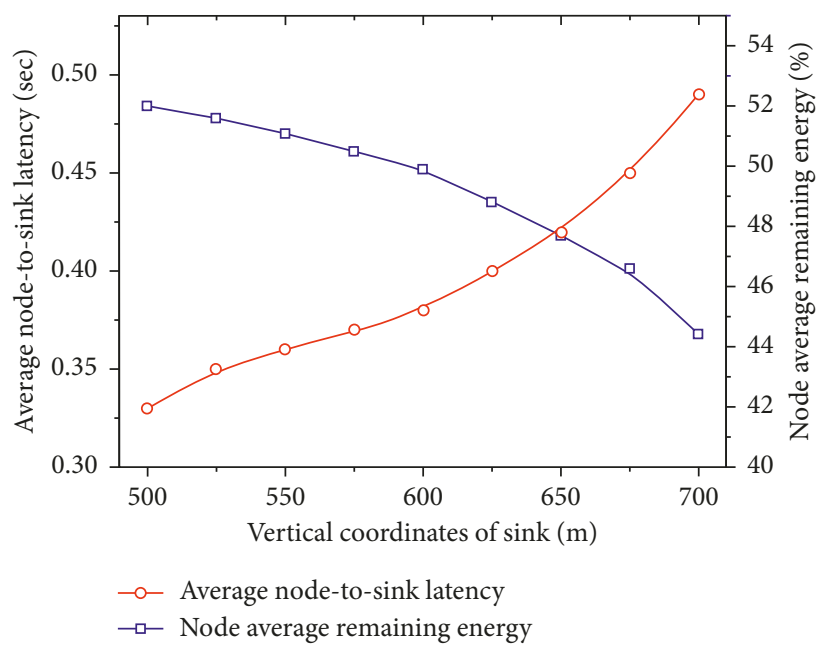

FIGURE 11: PB-MAC performance versus sink position changes.

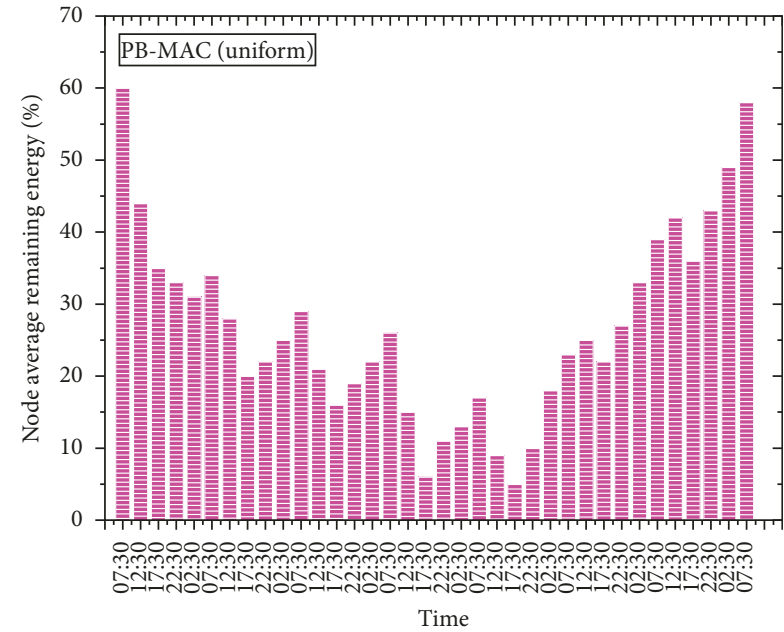

(a)

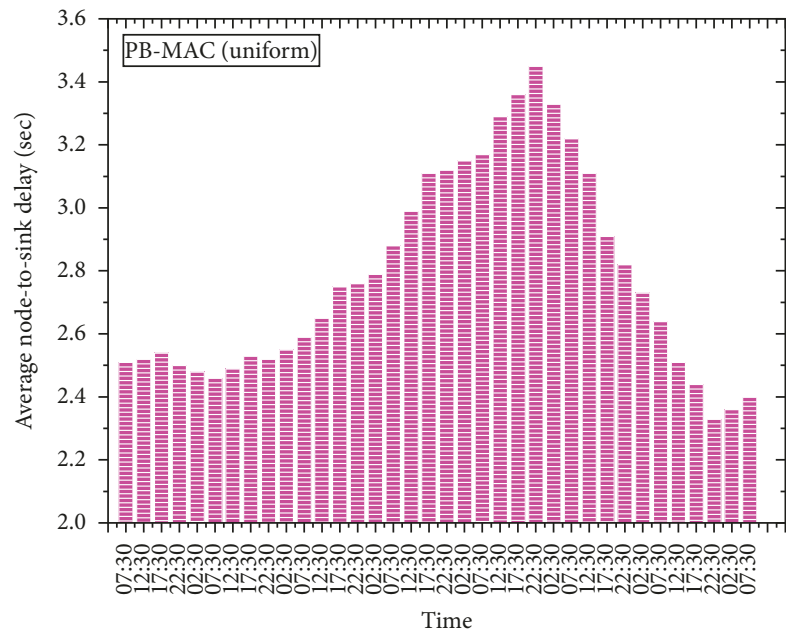

(c)

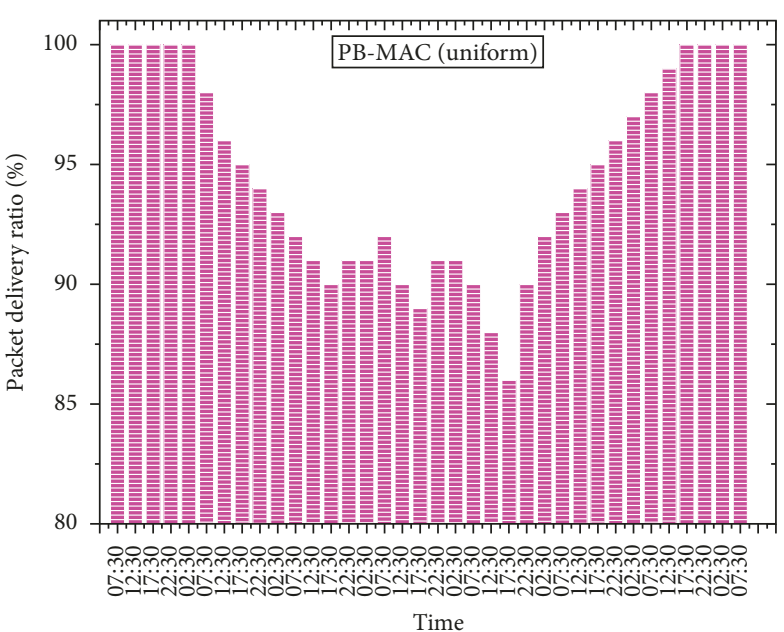

(b)

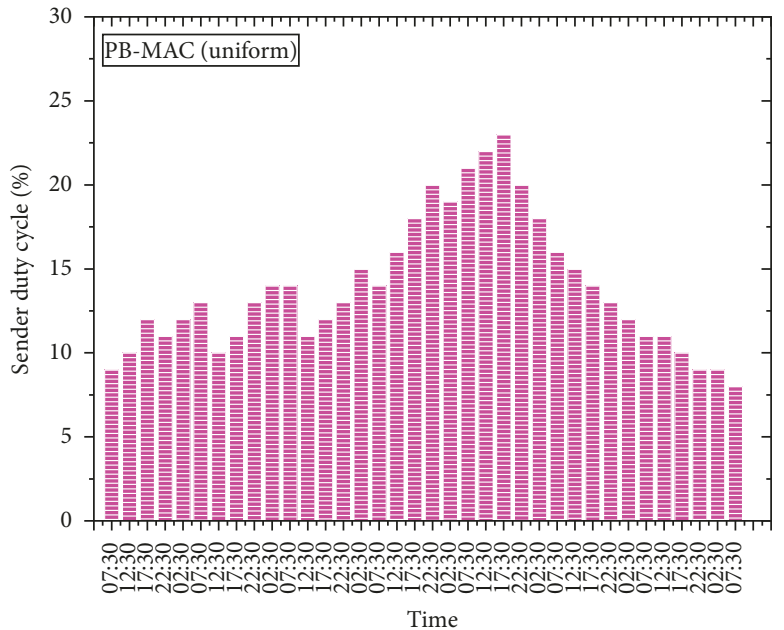

(d)

FiguRE 12: PB-MAC performance with 60\% initial energy level (uniform deployment). (a) Nodes' average remaining energy versus time of 7 days, (b) packet delivery ratio versus time of 7 days, (c) average node-to-sink latency versus time of 7 days, (d) sender duty cycle versus time of 7 days. 


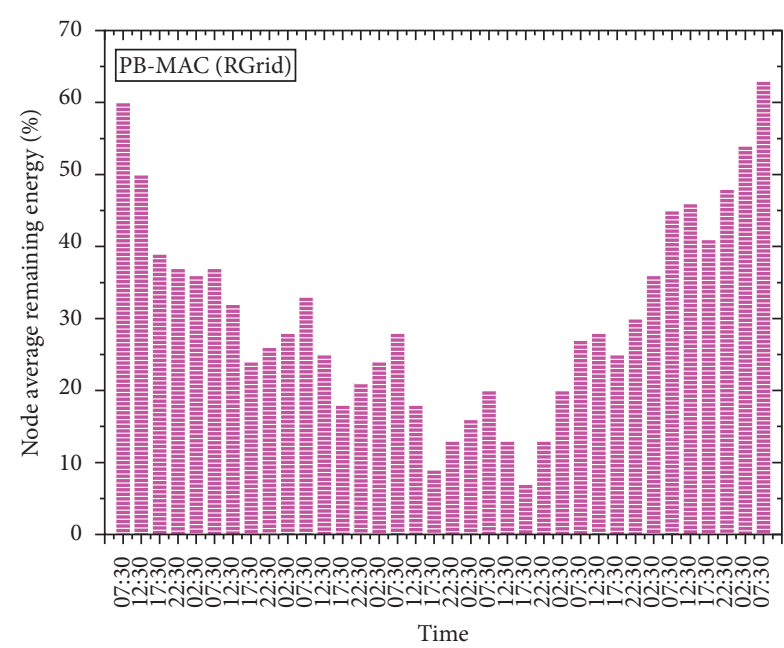

(a)

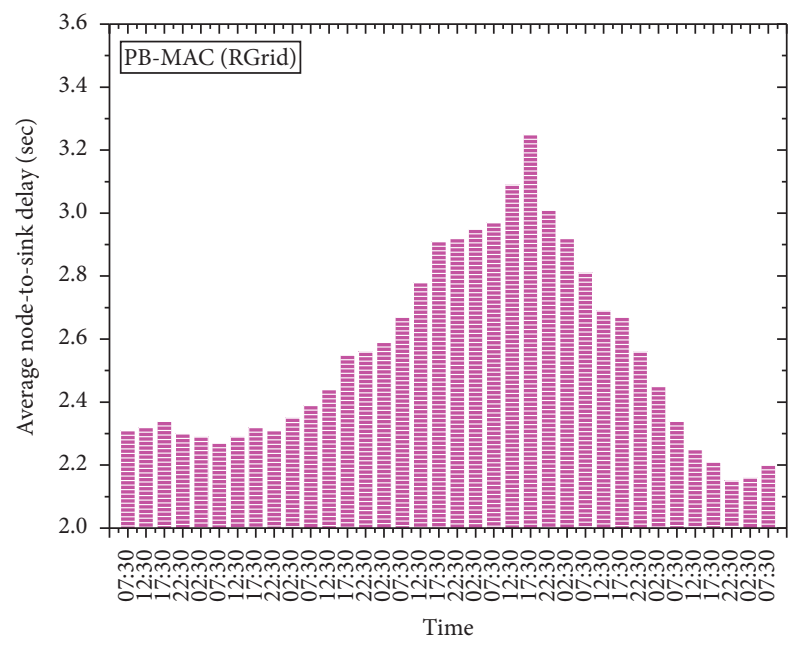

(c)

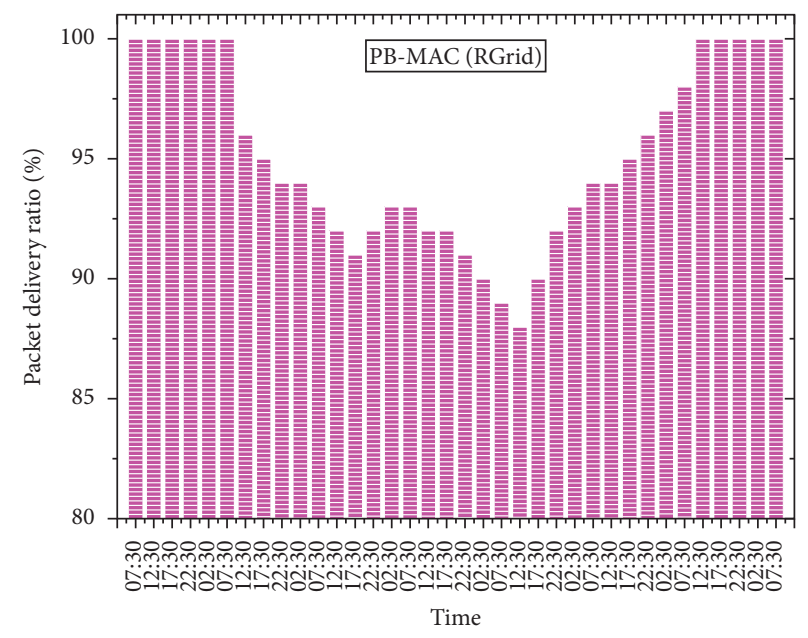

(b)

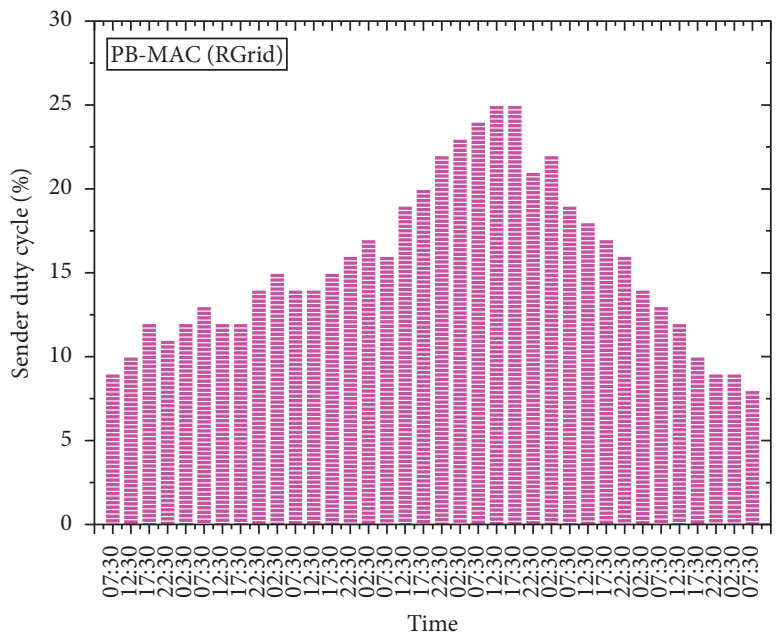

(d)

FIGURE 13: PB-MAC performance with 60\% initial energy level (RGrid deployment). (a) Nodes' average remaining energy versus time of 7 days, (b) packet delivery ratio versus time of 7 days, (c) average node-to-sink latency versus time of 7 days, (d) sender duty cycle versus time of 7 days.

Furthermore, we modify the nodes deployment to have an additional similar testing of PB-MAC. The network topology structure is set up referring to article [26] and an RGrid-based deployment is employed: all PBNs are randomly divided into 8 grids that revolve around the sink grid in the center. We also carry out the proposed grid-based multihop routing scheme, and four experiment results of a random chosen cluster member node are given in Figure 13.

Apparently, PB-MAC in RGrid shows better performance than the PBNs with uniform distribution in Figure 12. Especially the average node-to-sink latency in Figure 13(c) improves significantly: the reason is that the scheduling of grid-based transmission is in a preset order; PB-MAC takes full advantage of this distinction and saves considerable waiting time of idle listening. This also helps to enhance the node's average remaining energy (Figure 13(a)). As well, the other two indicators further demonstrate that PB-MAC has superior adaptability and survivability for different network topologies.
Thirdly, we compare the four protocols using empirical data for network density performance on a cloudy day with rain in the morning. Each PBN starts with a 50\% initial energy. The communication rate is $4 \mathrm{kB} / \mathrm{s}$ and the default packet size is 32 bytes. The experiment's result is shown in Figure 14.

As can be seen from the above diagram, data throughput rises along with the increased deployment of sensor nodes (Figure 14(a)). Particularly when the total number gets greater, average throughput of $\mathrm{PB}-\mathrm{MAC}$ grows faster than others do. It is caused by the accurate dynamic adjustment of work/sleep duty cycle, which improves the working duration optimally and keeps all PBNs energy neutrally. On the other side, when the number of nodes increases, we find that the PBNs' average remaining energy decreases gradually and the node-to-sink latency increases (Figures 14(b) and 14(c)), the reason is that more network nodes would induce more relays and therefore, the more energy consumption and transmission delay. However, PB-MAC can adapt to the variation of 


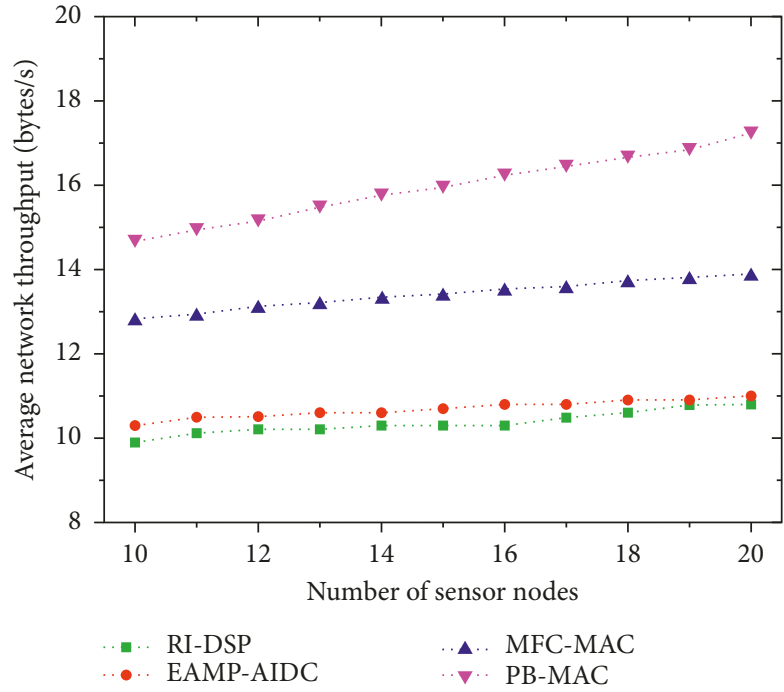

(a)

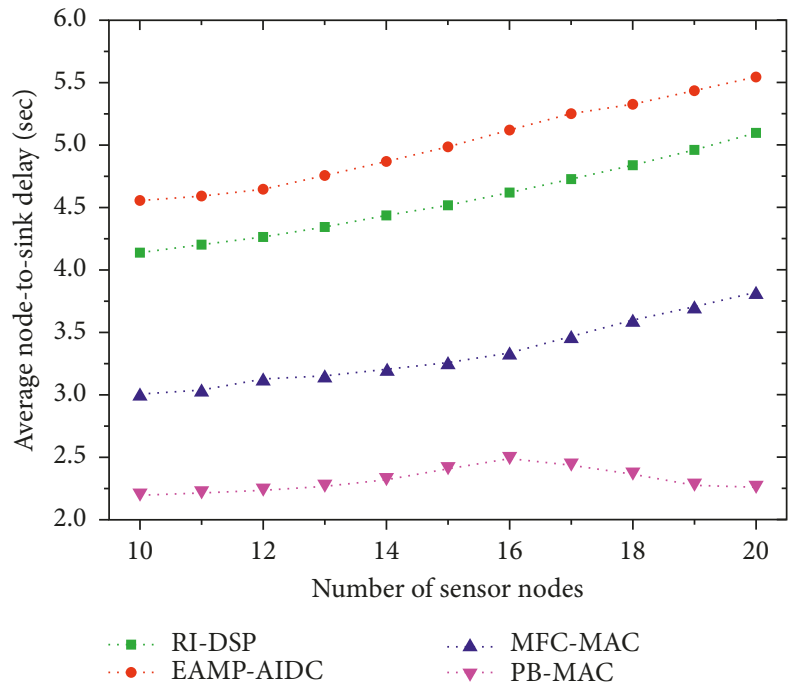

(c)

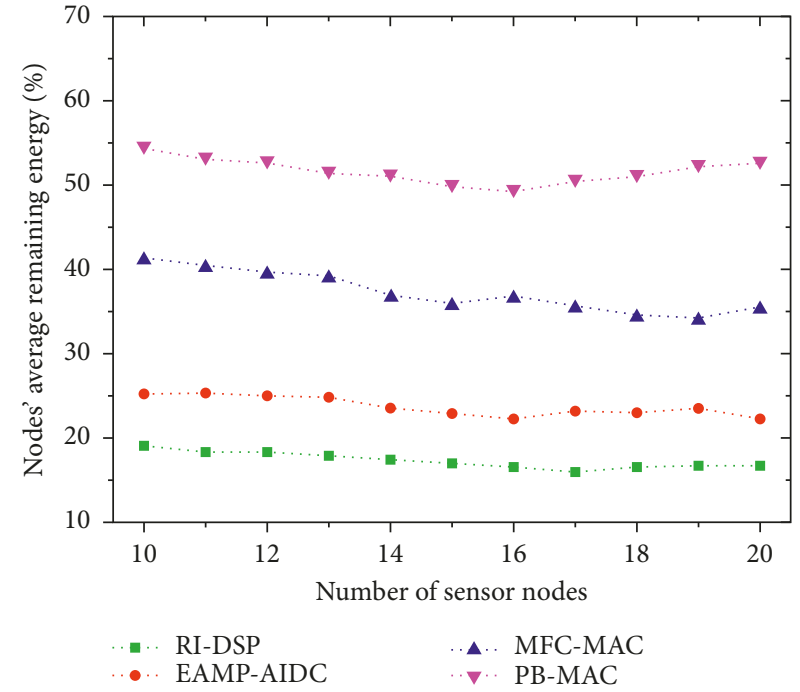

(b)

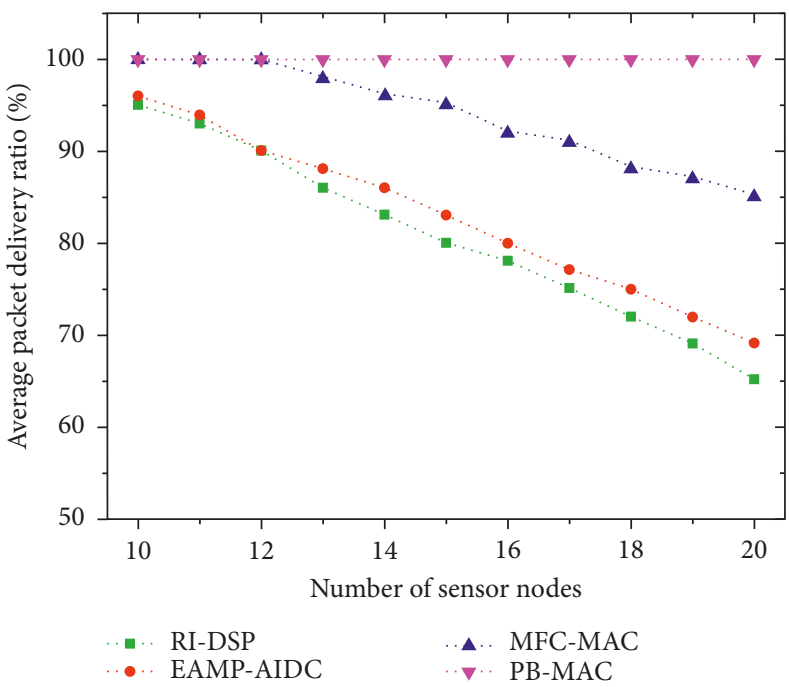

(d)

Figure 14: Node density performance comparison of four MAC protocols. (a) Average network throughput versus number of nodes, (b) nodes' average remaining energy versus number of nodes, (c) average node-to-sink latency versus number of nodes, (d) average packet delivery ratio versus number of nodes.

network topology and shows better scalability as for its highefficient channel access-scheduling ability. In addition, PDR of PB-MAC always maintains $100 \%$ while the others drop drastically as seen in Figure 14(d). Consequently, it can be deduced that PB-MAC has high performance and robustness when the node density changes.

Finally, we conduct the statistical inference of our approach: the experiment on PB-MAC, RI-DSP, EAMP-AIDC, and MFC-MAC has carried out in the mingled forest over 7 days. The average remaining energy (ARE) of the proposed PBNs have computed, and it forms a bivariate normal variable when pair wise-comparison is done between $\mathrm{PB}$ MAC and RI-DSP, or EAMP-AIDC, or MFC-MAC after every day. The pair of corresponding variables is in the form $\left(x_{i}, y_{i}\right)$ where $x_{i}$ is the ARE for PB-MAC and $y_{i}$ is the ARE for the existing protocol as stated above; these are corelated and should call for the use of paired T-test [27] for drawing statistical inference between the protocols being compared. The null and alternative hypotheses for the three cases are as follows:

(1) Null Hypothesis $H_{0}$ : $\left(\mathrm{ARE}_{\mathrm{PB}-\mathrm{MAC}}=\mathrm{ARE}_{\mathrm{RI}-\mathrm{DSP}}\right) \cdot \mathrm{Al}-$ ternative Hypothesis $H_{1}$ : $\left(\mathrm{ARE}_{\mathrm{PB}-\mathrm{MAC}}>\mathrm{ARE}_{\mathrm{RI}-\mathrm{DSP}}\right)$.

(2) Null Hypothesis $H_{0}^{\prime}$ : $\left(\mathrm{ARE}_{\mathrm{PB}-\mathrm{MAC}}=\mathrm{ARE}_{\mathrm{EAMP}-\mathrm{AIDC}}\right)$. Alternative Hypothesis $H_{1}^{\prime}$ : $\quad\left(\mathrm{ARE}_{\mathrm{PB}-\mathrm{MAC}}>\right.$ ARE $_{\text {EAMP-AIDC). }}$.

(3) Null Hypothesis $H_{0}^{\prime \prime}$ : $\left(\mathrm{ARE}_{\mathrm{PB}-\mathrm{MAC}}=\mathrm{ARE}_{\mathrm{MFC}-\mathrm{MAC}}\right)$. Alternative Hypothesis $H_{1}^{\prime \prime}: \quad\left(\mathrm{ARE}_{\mathrm{PB}-\mathrm{MAC}}>\right.$ $\mathrm{ARE}_{\mathrm{MFC}-\mathrm{MAC}}$ ).

The test statistic $t$ with $n-1$ degrees of freedom is defined as: $t=D_{\text {avg }} /\left[S_{\mathrm{d}} / \sqrt{(n-1)}\right]$, where $D_{\text {avg }}$ and $S_{\mathrm{d}}$ denote 
TABLE 2: Results of paired T-test.

\begin{tabular}{lcccc}
\hline \multirow{2}{*}{$\begin{array}{l}\text { Comparison } \\
\text { of PB-MAC }\end{array}$} & $\begin{array}{c}T \\
\text { value }\end{array}$ & $\begin{array}{c}\text { Significance } \\
\text { level }\end{array}$ & \multicolumn{2}{c}{$\begin{array}{c}\text { 95\% confidence } \\
\text { interval }\end{array}$} \\
& & & Lower & Upper \\
\hline RI-DSP & 42.25 & $95 \%$ & 44.21 & 48.65 \\
EAMP-AIDC & 33.21 & $95 \%$ & 31.71 & 35.87 \\
MFC-MAC & 21.67 & $95 \%$ & 6.59 & 7.91 \\
\hline
\end{tabular}

the mean and standard deviation of the difference of ARE in two equal sized correlated large samples of size $n$. The 95\% confidence limits for $D_{\text {avg }}$ is $D_{\text {avg }} \pm t_{0.05} *\left[S_{\mathrm{d}} / \sqrt{(n-1)}\right]$. Here $t_{0.05}$ is the $5 \%$ point of the $t$-distribution on $n-1$ degrees of freedom. Let $p$ indicate the probability of the calculated value for our test statistic $t$ with $n-1$ degrees of freedom to obey the null hypothesis. A value of $p<0.05$ indicates that $H_{0}, H_{0}^{\prime}$, and $H_{0}^{\prime \prime}$ is rejected at $5 \%$ significance level and hence $H_{1}, H_{1}^{\prime}$, and $H_{1}^{\prime \prime}$ be accepted at $95 \%$ confidence level.

Table 2 shows the result of paired $T$-test obtained by pair wise testing of PB-MAC with RI-DSP, EAMP-AIDC, and MFC-MAC, respectively. In all the cases $p<0.05$ so $H_{0}, H_{0}^{\prime}$, and $H_{0}^{\prime \prime}$ is rejected at $5 \%$ significance level and $H_{1}, H_{1}^{\prime}$, and $H_{1}^{\prime \prime}$ is accepted at $95 \%$ confidence level. In addition, the lower and upper limits for the $95 \%$ confidence interval for $D_{\text {avg }}$ are also listed. Hence, it can be convincingly concluded that PB-MAC outperforms RI-DSP, EAMP-AIDC, and MFC-MAC, and the results are statistically significant.

With all the scenarios studied, PB-MAC and MFCMAC are able to achieve much outstanding performance with shorter sender duty cycles. Between them, the former even better satisfies the plant living environment. Therefore, we can conclude that while all protocols are able to operate under good circumstance, only PB-MAC is able to continue superior operating under extremely low energy harvesting conditions.

\section{Conclusion and Future Work}

In this paper, a novel MAC protocol for sustaining perpetual operation of PB-WSN is proposed. Some relevant researches have been conducted: First, an individual energy harvesting prediction algorithm is proposed to guarantee precise energy management. Second, an optimized self-adaptive work/sleep duty cycle mechanism is introduced, aiming to provide intracluster wide optimal packet communications under extremely limited node resources. Finally, a priority-based communication method is employed to improve the intercluster wide transmission delay. Numerical simulations and actual experiment results are evaluated to analyze the performance of the proposed algorithm. Parameters such as the average node-to-sink latency, the sender's duty cycle ratio, the node average remaining energy, and the packet delivery ratio have been analyzed and reviewed. Results show the effectiveness and efficiency of the proposed MAC protocol.

Future work will focus on the proposal of cross layer optimization problem for PB-WSN and consider cognitive radio network issues.

\section{Conflicts of Interest}

The authors declare that there are no conflicts of interest regarding the publication of this paper.

\section{Acknowledgments}

This work is partially supported by the National Natural Science Foundation of China (nos. 31700478 and 31670554). The authors also gratefully acknowledge the helpful comments and suggestions of the reviewers, which have improved the presentation.

\section{References}

[1] F. Ongaro, S. Saggini, and P. Mattavelli, "Li-ion batterysupercapacitor hybrid storage system for a long lifetime, photovoltaic based wireless sensor network," IEEE Transactions on Power Electronics, vol. 27, no. 9, pp. 3944-3952, 2012.

[2] S. Lee, B. Kwon, S. Lee, and A. C. Bovik, "BUCKET: scheduling of solar-powered sensor networks via cross-layer optimization," IEEE Sensors Journal, vol. 15, no. 3, pp. 1489-1503, 2015.

[3] M. Shin and I. Joe, "Energy management algorithm for solarpowered energy harvesting wireless sensor node for internet of things," IET Communications, vol. 10, no. 12, pp. 15081521, 2016.

[4] R. Rana, W. Hu, and C. T. Chou, "Optimal sampling strategy enabling energy-neutral operations at rechargeable wireless sensor networks," IEEE Sensors Journal, vol. 15, no. 1, pp. 201-208, 2015.

[5] H. Yoo, M. Shim, and D. Kim, "Dynamic duty cycle scheduling schemes for energy-harvesting wireless sensor networks," IEEE Communications Letters, vol. 16, no. 2, pp. 202-204, 2012.

[6] T. N. Le, A. Pegatoquet, O. Berder, and O. Sentieys, "Energyefficient power manager and MAC protocol for multi-hop wireless sensor networks powered by periodic energy harvesting sources," IEEE Sensors Journal, vol. 15, no. 12, pp. 7208-7220, 2015.

[7] H.-I. Liu, W.-J. He, and W. K. G. Seah, "LEB-MAC: load and energy balancing MAC protocol for energy harvesting powered wireless sensor networks," in Proceedings of the IEEE International Conference on Parallel and Distributed Systems, pp. 584-591, Hsinchu, Taiwan, December 2014.

[8] O. Bouachir, A. Ben Mnaouer, F. Touati, and D. Crescini, "EAMP-AIDC-energy-aware MAC protocol with adaptive individual duty cycle for EH-WSN," in Proceedings of the 13th International IEEE Wireless Communications and Mobile Computing Conference (IWCMC), pp. 2021-2028, Valencia, Spain, June 2017.

[9] F. Yang, K.-C. Wang, and Y. Huang, "Energy-neutral communication protocol for very low power microbial fuel cell based wireless sensor network," IEEE Sensors Journal, vol. 15, no. 4, pp. 2306-2315, 2015.

[10] D. Zhang, F. Yang, T. Shimotori, K.-C. Wang, and Y. Huang, "Performance evaluation of power management systems in microbial fuel cell-based energy harvesting applications for driving small electronic devices," Journal of Power Sources, vol. 217 , pp. $65-71,2012$.

[11] C. Erbay, S. Carreon-Bautista, E. Sanchez-Sinencio, and A. Han, "High performance monolithic power management 
system with dynamic maximum power point tracking for microbial fuel cells," Environmental Science and Technology, vol. 48, no. 23, pp. 13992-13999, 2014.

[12] D. Brunelli, P. Tosato, and M. Rossi, "Flora health wireless monitoring with plant-microbial fuel cell," in Proceedings of the 30th Anniversary Eurosensors Conference-Eurosensors, pp. 1646-1650, Budapest, Hungary, September 2016.

[13] C. Konstantopoulos, E. Koutroulis, N. Mitianoudis, and A. Bletsas, "Converting a plant to a battery and wireless sensor with scatter radio and ultra-low cost," IEEE Transactions on Instrumentation and Measurement, vol. 65, no. 2, pp. 388398, 2016.

[14] Y. Wu and W. Liu, "Routing protocol based on genetic algorithm for energy harvesting-wireless sensor networks," IET Wireless Sensor Systems, vol. 3, no. 2, pp. 112-118, 2013.

[15] Y. Liu, Y. He, M. Li, J. Wang, K. Liu, and X. Li, "Does wireless sensor network scale? A measurement study on GreenOrbs," IEEE Transactions on Parallel and Distributed Systems, vol. 24, no. 10, pp. 1983-1993, 2013.

[16] D. P. Smith, G. G. Messier, and M. W. Wasson, "Boreal forest low antenna height propagation measurements," IEEE Transactions on Antennas and Propagation, vol. 64, no. 9, pp. 4004-4011, 2016.

[17] F. Zhang, I. Joe, D. Gao, and Y. Liu, "An efficient multiplecopy routing in intermittently connected mobile networks," International Journal of Future Generation Communication and Networking, vol. 9, no. 5, pp. 207-218, 2016.

[18] N. Michelusi, L. Badia, R. Carli, L. Corradini, and M. Zorzi, "Energy management policies for harvesting-based wireless sensor devices with battery degradation," IEEE Transactions on Communications, vol. 61, no. 12, pp. 4934-4947, 2013.

[19] F. Yang, D. Zhang, T. Shimotori, K.-C. Wang, and Y. Huang, "Study of transformer-based power management system and its performance optimization for microbial fuel cells," Journal of Power Sources, vol. 205, pp. 86-92, 2012.

[20] E. Y. A. Lin, J. M. Rabaey, and A. Wolisz, "Power-efficient rendez-vous schemes for dense wireless sensor networks," in Proceedings of the IEEE International Conference on Communications, pp. 3769-3776, Paris, France, June 2004.

[21] Q. Ying, W. Yuan, and N. Hu, "Improving the efficiency of harvesting electricity from living trees," Journal of Renewable and Sustainable Energy, vol. 7, no. 6, p. 063108, 2015.

[22] A. Tanaka, T. Ishihara, F. Utsunomiya, and T. Douseki, "Wireless self-powered plant health-monitoring sensor system," in Proceedings of the IEEE Sensors, pp. 1-4, Taipei, Taiwan, October 2012.

[23] Z. Hao, G. Wang, W. Li, J. Zhang, and J. Kan, "Effects of electrode material on the voltage of a tree-based energy generator," PLoS One, vol. 10, no. 8, article 0136639, 2015.

[24] Y. Kim, C. W. Park, and T.-J. Lee, "MAC protocol for energyharvesting users in cognitive radio networks," in Proceedings of the 8th International Conference on Ubiquitous Information Management and Communication, pp. 1-4, Siem Reap, Cambodia, January 2014.

[25] A. Goldsmith, Wireless Communications, Cambridge University Press, Cambridge, UK, 2005.

[26] A. Thakkar and K. Kotecha, "A new Bollinger band based energy efficient routing for clustered wireless sensor network," Applied Soft Computing, vol. 32, pp. 144-153, 2015.

[27] J. K. Sharma, Fundamentals of Business Statistics, Vikash Publishing House, Chennai, India, 2nd edition, 2014. 


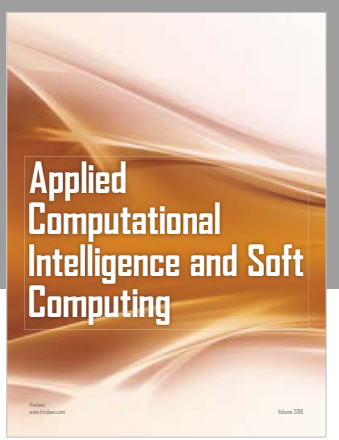

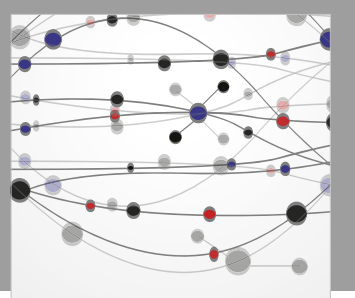

The Scientific World Journal
Submit your manuscripts at

Computing
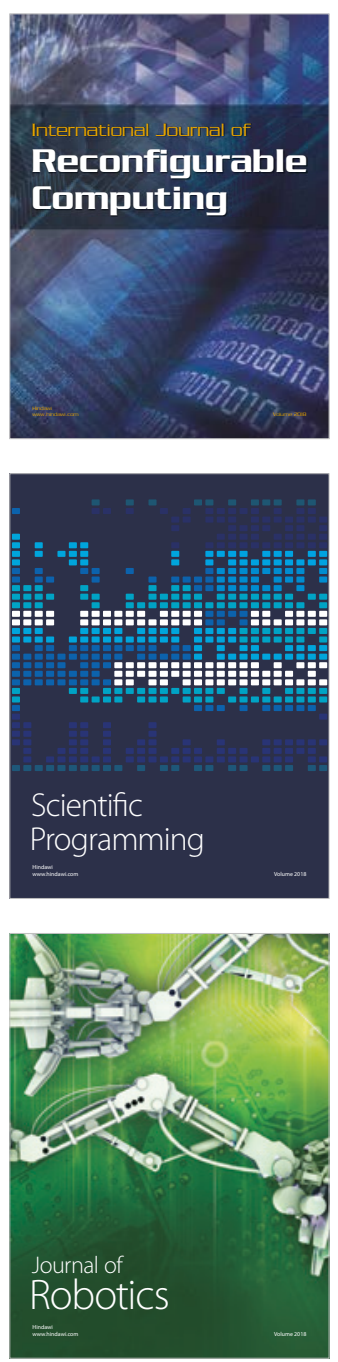

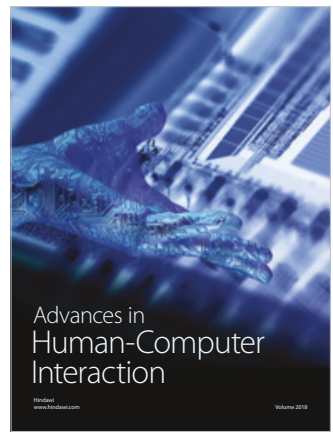

Human-Compute

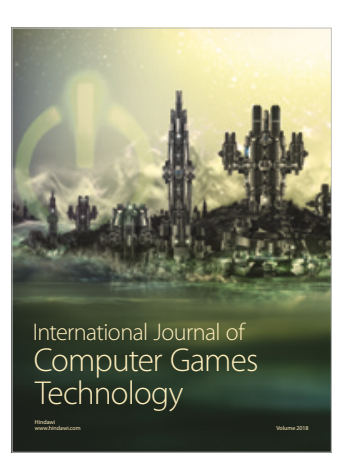

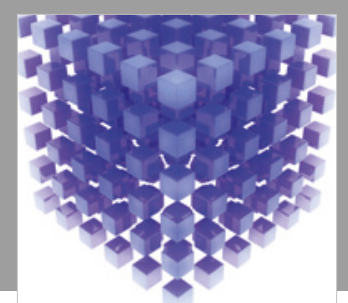

Mathematical Problems in Engineering

\section{Engincering}
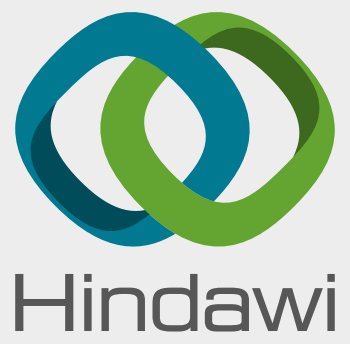

www.hindawi.com
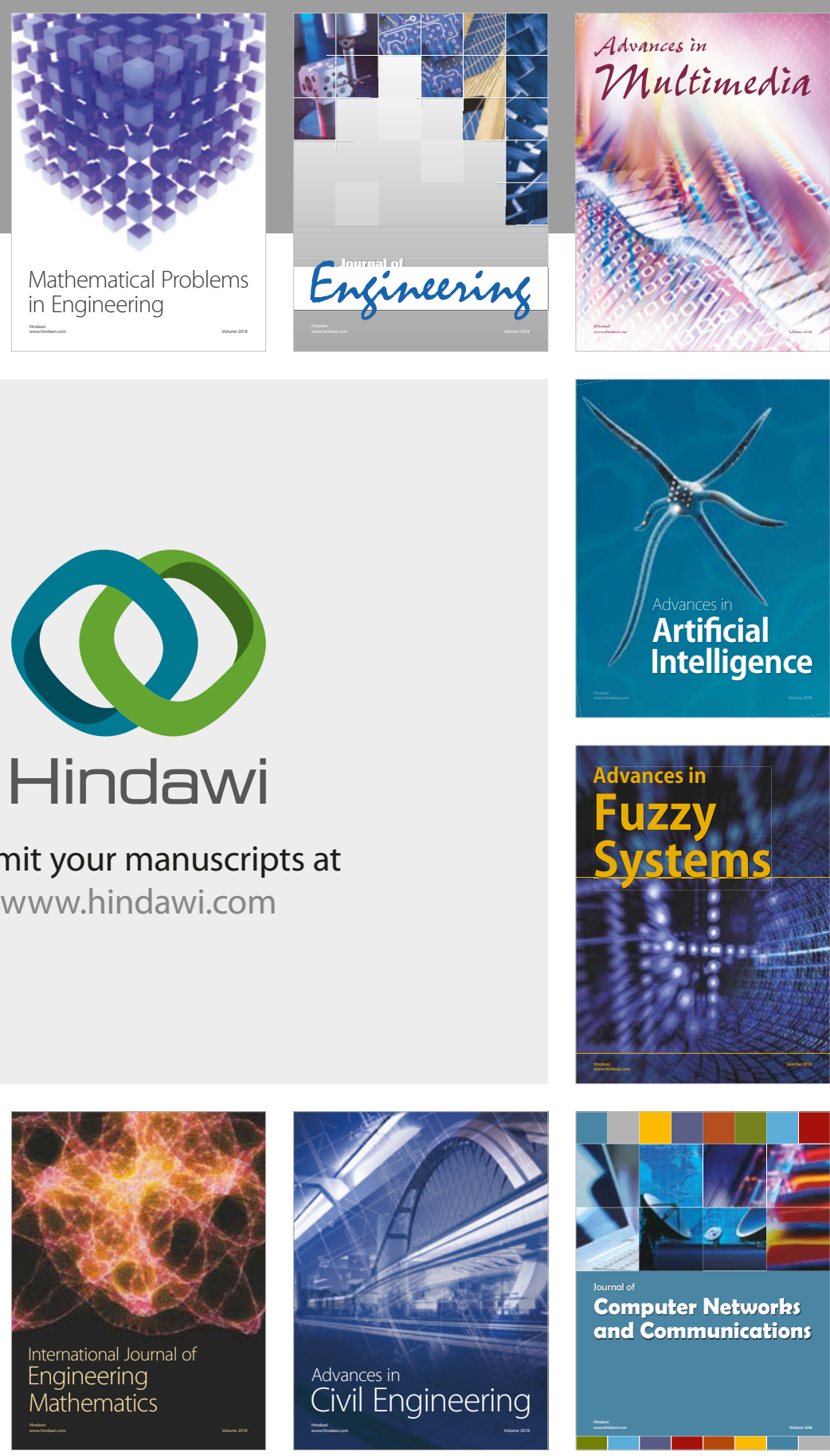

Computer Networks and Communications

Multimedia
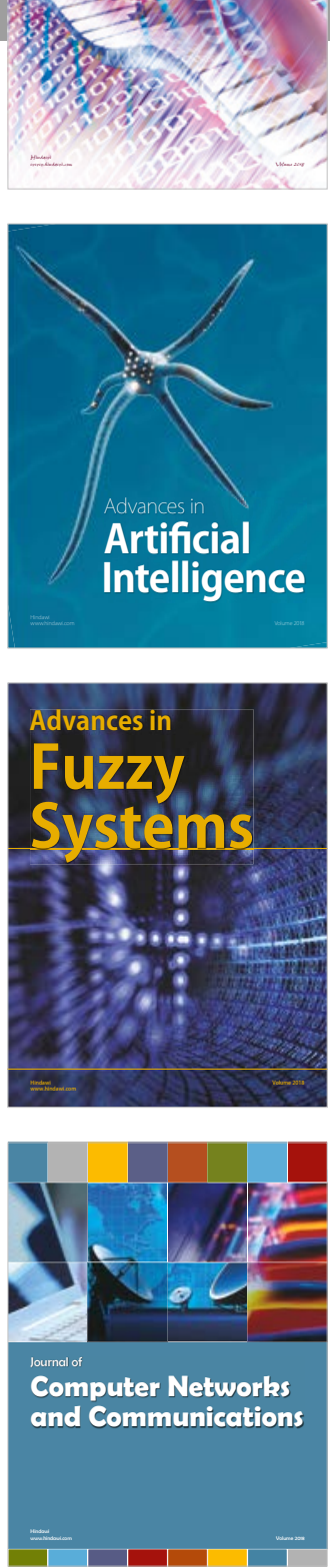

Advances in

Modelling \&

Simulation

in Engineering

interaction

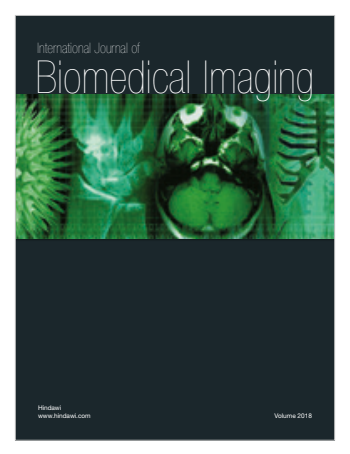

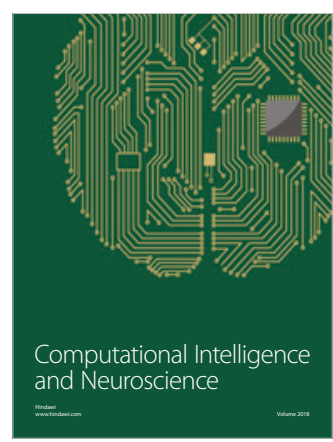

